\title{
Higher-level Inferences in the Strong-Kleene Setting: A Proof-theoretic Approach
}

\author{
Pablo Cobreros $^{1}$. Elio La Rosa ${ }^{2}$ - Luca Tranchini ${ }^{3}$ iD
}

Received: 16 June 2020 / Accepted: 21 September 2021 / Published online: 20 November 2021

(C) The Author(s) 2021

\begin{abstract}
Building on early work by Girard (1987) and using closely related techniques from the proof theory of many-valued logics, we propose a sequent calculus capturing a hierarchy of notions of satisfaction based on the Strong Kleene matrices introduced by Barrio et al. (Journal of Philosophical Logic 49:93-120, 2020) and others. The calculus allows one to establish and generalize in a very natural manner several recent results, such as the coincidence of some of these notions with their classical counterparts, and the possibility of expressing some notions of satisfaction for higher-level inferences using notions of satisfaction for inferences of lower level. We also show that at each level all notions of satisfaction considered are pairwise distinct and we address some remarks on the possible significance of this (huge) number of notions of consequence.
\end{abstract}

Keywords Higher-level rules · Labelled sequent calculus · Three-valued logic . Meta-inference $\cdot$ Paraconsistency $\cdot$ Paracompleteness

\section{Introduction}

Three-valued semantic settings make room for the distinction between the truth and the non-falsity of formula in a valuation. Famously, the distinction has been used

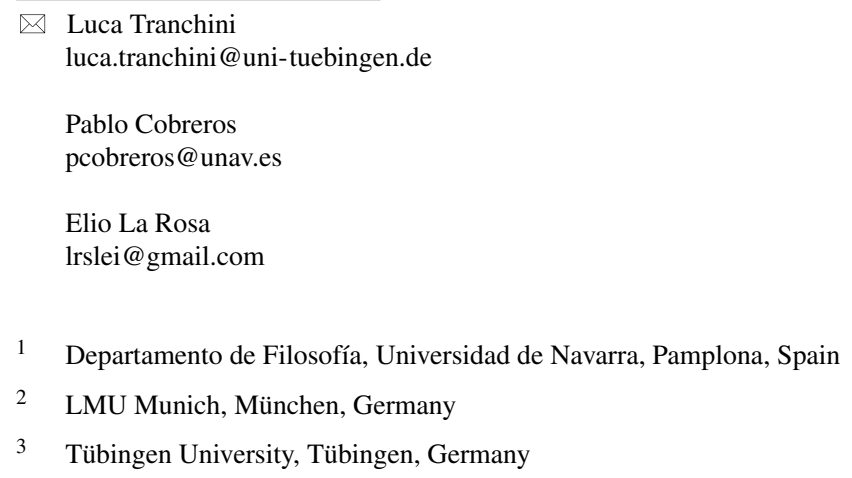


to define - given the same way of assigning truth-values to formulas - two different notions of validity of an inference: both the logic K3 and LP are based on the Strong Kleene (henceforth SK) matrices, but whereas the K3-validity of an inference $A \Rightarrow B$ is defined as the preservation of truth, its LP-validity is defined as the preservation of non-falsity from $A$ to $B$. Dubbing truth and non-falsity (respectively) as strict and tolerant satisfaction, [6] showed how, by adopting different standards of satisfaction between premises and conclusions, one can define two further "mixed" notions of validity of inferences in the SK-setting: ST-validity (in every valuation in which the premise is strictly satisfied, the conclusion must be tolerantly satisfied as well) and TS-validity (in every valuation in which the premise is tolerantly satisfied, the conclusion must be strictly satisfied as well).

Whereas the tolerant validity of a formula (i.e., its tolerant satisfaction in all valuations) coincides with its classical validity, it is not the case that preservation of tolerant satisfaction in all valuations coincides with the classical validity of an inference. Surprisingly, the latter coincides with ST-validity. ${ }^{1}$ Dually, whereas the set of strictly valid formulas is empty, it is the set of TS-valid inferences (and not that of K3-valid inferences) that is empty.

In spite of ST's "classicality" at the level of inferences, it has been observed that ST fails to validate some classical meta-inferences, i.e. expressions of the form $\Gamma \Rightarrow$ $\Delta$ in which $\Gamma$ and $\Delta$ do not contain formulas, but inferences. A meta-inference is said to be ST-valid iff whenever all inferences in $\Gamma$ are ST-satisfied in a valuation, so is at least one of the inferences in $\Delta$ [9]. Typical examples of meta-inferences which are not ST-valid are instances of "the transitivity of the inferential arrow", such as: $(P \Rightarrow Q),(Q \Rightarrow R) \Rightarrow(P \Rightarrow R)$. Actually, as shown by [2], a meta-inference $\Gamma \Rightarrow \Delta$ is ST-valid iff what [9] calls its lowering (i.e., the result of replacing $\Rightarrow$ with $\Rightarrow$ and each inference $\Sigma \Rightarrow \Theta$ in both $\Gamma$ and $\Delta$ with the formula $\wedge \Sigma \supset \bigvee \Delta$ ) is an LP-valid inference, a fact sometimes referred to as ST being LP in sheep's clothes.

As we recalled above, the adoption of different standards of satisfaction between premises and conclusions allows us to define a notion of validity of inferences that coincides with classical validity. As shown by [3], the same happens for metainferences: a meta-inference $\Gamma \Rightarrow \Delta$ is classically valid iff in every valuation in which all inferences in $\Gamma$ are TS-satisfied, at least one inference in $\Delta$ is ST-satisfied. [17] and [3] moreover show how this phenomenon can be iterated, by considering "meta-meta-inferences", "meta-meta-meta-inferences" and so on, and by defining for each of these notion a "mixed" notion of validity coinciding with the corresponding classical notion.

These results have been "dualized" by [22] who defined a hierarchy of notions of unsatisfiability for meta-...-meta-inferences, each of which coincides with an

\footnotetext{
${ }^{1}$ This was in fact already observed in the '70s by Schütte, who used this fact to provide a semantic proof of cut-elimination for second-order logic.
} 
appropriate generalization of the classical notion of unsatisfiability to meta-... -metainferences.

All this seems to call for a general framework encompassing all these notions and in which these results can be presented in a uniform fashion. In the present article we propose a possible formulation of such a framework, mainly relying on tools developed in the field of the proof theory of many-valued logics. As we will show, this framework allows one to reprove and generalize several of the above results and to establish new ones in a very natural and straightforward way. We therefore hope that the proposed framework can help in getting a clearer grasp of the structure of the SK-semantic setting, and can set the stage for further investigations.

The plan of the paper is as follows. In Section 2 we introduce the SK-semantic setting by recalling the notions of strict and tolerant satisfaction, as well as the four notions of satisfaction of an inference definable in their terms. We stress that these notions come equipped with a natural ordering between them, and recall how the classical notions of satisfaction of formulas and inferences can be recovered in this setting.

In Section 3 we present in an accessible way the sequent calculus G3SKessentially due to [12] — capable of capturing in a syntactic fashion the semantic notions previously introduced. Thereby, we hope to bring to the attention of the philosophical community at large Girard's essential contribution to the understanding of the SK-setting (which has been otherwise rather neglected in current literature, with the notable exception of [9]). Girard's system actually predates later development in the proof theory of many valued logics that we detail separately in Section 10. In Section 3 we clarify the relationship between G3SK and the standard sequent calculus for classical logic G3C by introducing unlabeling and labeling procedures allowing to go back and forth between the two systems.

After briefly introducing the notion of higher-level inference in Section 4, in Section 5 we first show how the classical notion of satisfaction can be scaled so as to apply to inferences of any level, and then how the sequent calculus G3C can be generalized to a calculus $\mathrm{G} \mathrm{C}^{\omega}$ capable of capturing all these semantically defined notions. In Section 6 we generalize in an analogous manner the SK-semantic framework to obtain a hierarchy of SK-notions of satisfaction for inferences of any level, and in Section 7 we generalize the sequent calculus G3SK to the calculus G3SK ${ }^{\omega}$ capturing the $\mathrm{SK}^{\omega}$-semantic framework by proof-theoretic means.

In Section 8 we establish a relationship between $S^{\omega}{ }^{\omega}$-notions of validity of different levels by scaling the notion of lowering to the whole $\mathrm{SK}^{\omega}$-setting. We moreover show how to scale the notions of (un)labeling introduced in Section 3 to the whole SK ${ }^{\omega}$ setting and use them to relate the classical calculus $\mathrm{G} \mathrm{C}^{\omega}$ to the calculus $\mathrm{G} 3 \mathrm{SK}^{\omega}$.

Besides detailing the connection with the proof-theoretic study of many-valued logics, in Section 10 we briefly discuss another proof-theoretic tool related to the proposed framework: the systems of nested sequents.

In Section 11 we give some philosophical comments on the results presented and hint at further directions of research. 


\section{SK-semantics for Formulas and Inferences}

Given a set $\mathcal{V}$ of propositional variables $P, Q, R, \ldots$ (possibly indexed) we consider the propositional language ${ }^{2} \mathcal{L}$ defined by the following grammar:

$$
A::=\mathcal{V}|\top| \perp|\neg A|(A \wedge A)|(A \vee A)|(A \supset A) \text {. }
$$

Outermost parentheses will mostly be omitted. We call a formula structural iff it contains no occurrences of $\top$ and $\perp$. An atomic formula (or simply atom) is an element of $\mathcal{V} \cup\{\top, \perp\}$.

\subsection{Strict, Tolerant and Classical Validity of Formulas}

We call any function $v: \mathcal{V} \mapsto\left\{1, \frac{1}{2}, 0\right\}$ an SK-valuation or simply valuation. The truth-value of a formula $A$ in a valuation $v$, indicated with $\llbracket A \rrbracket_{v}$, is defined as follows:

$$
\begin{array}{ll}
\llbracket P \rrbracket_{v}=v(P) \text { for } P \in \mathcal{V} & \llbracket \top \rrbracket_{v}=1 \quad \llbracket \perp \rrbracket_{v}=0 \\
\llbracket A \wedge B \rrbracket_{v}=\min \left(\llbracket A \rrbracket_{v}, \llbracket B \rrbracket_{v}\right) & \llbracket A \vee B \rrbracket_{v}=\max \left(\llbracket A \rrbracket_{v}, \llbracket B \rrbracket_{v}\right) \\
\llbracket \neg A \rrbracket_{v}=1-\llbracket A \rrbracket_{v} & \llbracket A \supset B \rrbracket_{v}=\max \left(1-\llbracket A \rrbracket_{v}, \llbracket B \rrbracket_{v}\right)
\end{array}
$$

Following [6], we say that a formula $A$ is strictly satisfied by a valuation $v$, written $v \vDash_{\mathrm{s}} A$, when $\llbracket A \rrbracket_{v}=1$ and that it is tolerantly satisfied, written $v \vDash_{\mathrm{t}} A$, when $\llbracket A \rrbracket_{v}>0$. A formula is strictly valid (resp. tolerantly valid), $\models_{\mathrm{s}} A\left(\vDash_{\mathrm{t}} A\right)$, iff it is strictly satisfied (tolerantly satisfied) by all valuations. A formula is strictly unsatisfiable (resp. tolerantly unsatisfiable), $A \vDash_{\mathrm{s}}\left(A \vDash_{\mathrm{t}}\right)$, iff it is not strictly satisfied (tolerantly satisfied) by any valuation.

Observe that if a valuation $v$ maps no propositional variable on $\frac{1}{2}$, then the truthvalue of any formula $A$ in $v$ is either 0 or 1 . Such valuations will be called classical, or shortly $\mathrm{C}$-valuations. ${ }^{3}$ Clearly, for $\mathrm{C}$-valuations tolerant and strict satisfaction collapse, i.e. $v \vDash_{\mathrm{s}} A$ iff $v \vDash_{\mathrm{t}} A$, and in this case we will say that $v \mathrm{C}$-satisfies (or simply satisfies) $A$. We say that $A$ is classically valid, or C-valid, notation $\vDash A$, iff it is satisfied by all $\mathrm{C}$-valuations. To single out the strict and tolerant notions of satisfaction (resp. validity), we will refer to them as SK-notions of satisfaction (validity).

In general, however, strict satisfaction implies tolerant satisfaction but not the other way around. Moreover, tolerant validity is implied but does not imply strict validity, and dually strict unsatisfiability is implied but does not imply tolerant unsatisfiability. That is, the set of strictly valid formulas is a proper subset of the set of tolerantly valid formulas and the set of tolerantly unsatisfiable formulas is a proper subset of the set of strictly unsatisfiable formulas.

\footnotetext{
${ }^{2}$ For reasons of simplicity, we will not consider quantified extensions, although all results below could be extended to the first-order setting in a straightforward manner.

${ }^{3}$ Also, observe that the truth-value of the constant formulas we consider (i.e., $\top$ and $\perp$ ) is either 0 or 1 in any SK-valuation.
} 
Remark 1 In fact, the set of strictly valid structural formulas is empty and the set of tolerantly valid formulas coincides with that of C-valid formulas. Dually, the set of strictly unsatisfiable formulas coincides with that of C-unsatisfiable formulas, and the set of tolerantly unsatisfiable structural formulas is empty. ${ }^{4}$ Although these facts may not be obvious from our semantic definitions, we will provide a simple argument for them in Section 3 (see in particular, Section 3.2).

Remark 2 Let ${ }^{-}$be the function mapping t to $s$ and $s$ to t. Strict and tolerant satisfaction are duals in the sense that $v \nvdash_{\mathrm{x}} A$ iff $v \vDash_{\overline{\mathrm{x}}} \neg A$ (and hence $A \vDash_{\mathrm{x}}$ iff $\not \nvdash_{\overline{\mathrm{x}}} \neg A$ ). For C-valuations, satisfaction is self-dual. (For a discussion of different notions of duality in the $\mathrm{SK}^{\omega}$-setting, see [7].)

\subsection{Satisfaction and validity of inferences}

Let $\Gamma$ and $\Delta$ be finite (possibly empty) multisets of formulas. We call inference any expression of the form ( $\Gamma \Rightarrow \Delta$ ) (outer parenthesis will often be omitted), and we call $\Gamma$ the antecedent and $\Delta$ the succedent. An inference $\Gamma \Rightarrow \Delta$ is structural iff at least one among $\Gamma$ and $\Delta$ is non empty and all formulas in $\Gamma$ and $\Delta$ are structural.

Given the two SK-notions of formula satisfaction, we can define four different SK-notions of inference satisfaction as follows (here and below we use w, x, y, z as variables ranging over $t$ and $s$ ):

$$
v \vDash_{\mathrm{xy}}(\Gamma \Rightarrow \Delta) \text { iff if } v \vDash_{\mathrm{x}} A \text { (for all } A \in \Gamma \text { ) then } v \vDash_{\mathrm{y}} B \text { (for some } B \in \Delta \text { ) }
$$

We say that $\Gamma \Rightarrow \Delta$ is xy-valid, written $\vDash_{x y}(\Gamma \Rightarrow \Delta)$, iff it is xy-satisfied by every valuation, and that it is $x y$-unsatisfiable, written $(\Gamma \Rightarrow \Delta) \vDash_{x y}$, iff it is not xy-satisfied by any valuation. 5,6

\footnotetext{
${ }^{4}$ The qualification "structural" here and in the previous sentence is essential, since obviously $\top$ is strictly valid and $\perp$ is tolerantly unsatisfiable.

${ }^{5}$ The definitions of the SK-notions of validity of an inference are "local", i.e., these notions have been defined from the notion of satisfaction of an inference by a valuation by prefixing it with a universal quantification over all valuations. Alternative "global" notions of validity of an inference can be obtained, e.g., in the case of the SK-notions of validity of an inference, from the following scheme:
}

$$
\vDash_{\mathrm{xy}} \Gamma \Rightarrow \Delta \text { iff } \vDash_{\mathrm{x}} A \text { (for all } A \in \Gamma \text { ) then } \vDash_{\mathrm{y}} B \text { (for some } B \in \Delta \text { ) }
$$

where both the antecedent and the consequent of the conditional to the right of the 'iff' are validity (and not satisfaction) claims, i.e., they consist of a universal quantification over all valuations. The "swap" between the quantifiers makes a lot of difference. For instance on the global definition, the inference $P \Rightarrow Q$ would be valid on any choice of $x$ and $y$. The global notions of validity of an inference are therefore at odds with any intuitive notion of validity of an inference (which, to us quite uncontroversially, does not allow one to infer any propositional variable from any other). We therefore chose a local definition of validity of an inference. Similar reasons will motivate local definitions of validity for inferences of higher-level in Section 4. For the significance of global notions see, e.g., [8].

${ }^{6}$ In contrast to what happens for validity, in the case of unsatisfiability, the following "global" definition

$$
\Gamma \Rightarrow \Delta \vDash_{\mathrm{xy}} \text { iff } \vDash_{\mathrm{x}} A \text { (for all } A \in \Gamma \text { ) and } B \vDash_{\mathrm{y}} \text { (for all } B \in \Delta \text { ) }
$$

is equivalent to the local one. 
Fig. 1 The relationship between the notions of inference satisfaction



Remark 3 The four SK-notion of inference validity coincide respectively with the notions of validity of an inference adopted in the logics known in the literature as Strong Kleene logic (K3) for $x=y=s$, Logic of Paradox (LP) for $x=y=t$, StrictTolerant logic (ST) for $\mathrm{x}=\mathrm{s}$ and for $\mathrm{y}=\mathrm{t}$ and Tolerant-Strict logic (TS) for $\mathrm{x}=\mathrm{t}$ and for $y=s$. In the following we will use K3 and ss interchangeably, and similarly for LP, ST, TS.

As for C-valuations tolerant and strict satisfaction collapse, so do the four notions of inference satisfaction, i.e., for all C-valuations $v, v \vDash_{\mathrm{SS}} \Gamma \Rightarrow \Delta$ iff $v \vDash_{\mathrm{tt}} \Gamma \Rightarrow \Delta$ iff $v \vDash_{\mathrm{ts}} \Gamma \Rightarrow \Delta$ iff $v \vDash_{\mathrm{st}} \Gamma \Rightarrow \Delta$, and in this case we say that $\Gamma \Rightarrow \Delta$ is Csatisfied, or simply satisfied by $v$. We say that an inference is classically valid, or C-valid, notation $\vDash \Gamma \Rightarrow \Delta$, iff it is satisfied by all C-valuations, and similarly for C-unsatisfiable.

The relationship between tolerant and strict satisfaction of formulas induces a (partial) order between the four SK-notions of inference satisfaction, the Hasse diagram of which is given in Fig. 1. More "tolerant" notions of satisfaction are obtained either by setting more tolerant standards of satisfaction in the succedent or "stricter" standards of satisfaction in the antecedent of the inference.

The order is strict and actually for any choice of $x, y, w, z$, if wz-satisfaction is more tolerant than $x y$-satisfaction, then there are wz-valid inference which are not $x y$-valid and there are $x y$-unsatisfiable inferences that are not wz-unsatisfiable.

Example 1 The inference $\Rightarrow A \vee \neg A$ is tt- (and hence st-) valid but neither ts- nor ss-valid, and $A \wedge \neg A \Rightarrow$ is ss- (and hence st-) valid but neither ts-nor tt-valid. Dually, the inference $A \vee \neg A \Rightarrow$ is tt- (and hence ts-) unsatisfiable but neither st- nor ssunsatisfiable, and $\Rightarrow A \wedge \neg A$ is ss- (and hence ts-) unsatisfiable but neither st- nor tt-unsatisfiable.

Remark 4 In fact, the set of TS-valid structural inferences ${ }^{7}$ is empty and the set of ST-valid inferences coincides with that of C-valid inferences. As for Remark 1, although these facts may not be obvious from our semantic definitions, we will provide a simple argument for them in the next section (see, in particular, Section 3.2).

\footnotetext{
${ }^{7}$ The qualification is necessary for reasons analogous to those mentioned in footnote 4 .
} 


\section{Proof Theory of SK-inferences}

Proof-theoretic investigations of many-valued logics (of which the SK-semantic setting is a prominent example) have a long tradition dating back at least to the '50s (see $[20,25])$ and today constitute one of the major research directions in the field of non-classical logic. Independently of this tradition, the SK-semantic setting has been investigated in the early '60s by Schütte and Tait, who used it to establish the cut-elimination theorem for second-order logic (Takeuti's conjecture) by semantic means. This line of research has been further pursued by Girard in the '70s [12, Ch. 3]. In particular, Girard proposed to capture the SK-semantic setting using a labeled sequent calculus, which we present in this section in a slightly modified form that will allow a more straightforward presentation of some of the results below.

The connections between the work of Girard in the Tait-Schütte tradition on the SK-setting and the more general investigations in the proof theory on many-valued logics will be reviewed in Section 10, and the reader familiar with the latter tradition is invited to consult that Section already now.

\subsection{The Calculus G3SK}

A labeled formula is an expression of the form $\mathrm{x}: A$, for $A \in \mathcal{L}$ and $\mathrm{x} \in\{\mathrm{s}, \mathrm{t}\}$. A labeled formula $\mathrm{x}$ : $A$ is structural if $A$ is.

Remark 5 To avoid unnecessary proliferation of fonts, we use $\Gamma, \Delta, \ldots$ both for finite multisets of formulas and of labeled formulas, the context always making clear which is meant. Moreover, when $\Gamma$ is a multiset of formulas, we indicate with $\mathrm{x}: \Gamma$ the multiset of labeled formulas $\{\mathrm{x}: A \mid A \in \Gamma\}$. We will use $\Phi$ and $\Psi$ for multisets of labeled atoms, i.e., expressions $\mathrm{x}: A$ with $A \in \mathcal{V} \cup\{\top, \perp\}$.

An SK-sequent is an expression of the form $\Gamma \triangleright \Delta$ in which $\Gamma$ and $\Delta$ are (finite, possibly empty) multisets of labeled formulas called the antecedent and the succedent respectively. An SK-sequent is structural (resp. constant) iff at least one among $\Gamma$ and $\Delta$ are non-empty and all labeled formulas in $\Gamma$ and $\Delta$ are structural. The general form of an SK-sequent can thus be depicted as follows:

$$
\mathrm{s}: \Gamma_{1}, \mathrm{t}: \Gamma_{2} \triangleright \mathrm{s}: \Delta_{1}, \mathrm{t}: \Delta_{2}
$$

(where $\Gamma_{1}, \Gamma_{2}, \Delta_{1}, \Delta_{2}$ are possibly empty). Semantically, we say that an SK-sequent is satisfied by an SK-valuation $v$ iff if $v \vDash_{\mathrm{S}} A$ for all $A \in \Gamma_{1}$ and $v \vDash_{\mathrm{t}} B$ for all $B \in \Gamma_{2}$, then either $v \vDash_{\mathrm{s}} C$ for some $C \in \Delta_{1}$ or $v \vDash_{\mathrm{t}} D$ for some $D \in \Delta_{2}$. We say that an SK-sequent is valid iff it is satisfied by all valuations.

Remark 6 When both one among $\Gamma_{1}$ or $\Gamma_{2}$ and one among $\Delta_{1}$ or $\Delta_{2}$ are empty, the satisfaction/validity of an SK-sequent coincides with the satisfaction/validity of the corresponding inference according to one of the SK-notions of satisfaction/validity of an inference. For instance, if $\Gamma_{1}$ and $\Delta_{2}$ are empty we have an SK-sequent of the form $\mathrm{t}: \Gamma_{2} \triangleright \mathrm{s}: \Delta_{1}$, and such a sequent is satisfied by $v$ iff the inference $\Gamma_{2} \Rightarrow \Delta_{1}$ is ts-satisfied by $v$. Observe however that SK-sequents and inferences are 
Table 1 The rules of G3SK



distinct linguistic entities (the former contain labeled formulas, the latter just formulas). Although this apparently unnecessary duplication may be irritating for the reader at this point, in the following section it will enable us to develop a prooftheoretic account of inferences of higher-level in a smooth manner. To stress the connection between the satisfaction of an SK-sequent of the form $x: \Gamma \triangleright y: \Delta$ and the xy-satisfaction of $\Gamma \Rightarrow \Delta$ we will refer to sequents of this form as xy-sequents.

We also remark that the $x$-satisfaction/x-validity (respectively non-x-satisfaction/x-unsatisfiability) of a formula $A$ are equivalent to the satisfaction/validity of the sequent $\triangleright \mathrm{x}: A$ (resp. $\mathrm{x}: A \triangleright)$.

A G3SK-derivation, or shortly an SK-derivation, is a tree of SK-sequents constructed according to the propositional rules and whose leaves are the initial sequents given in Table 1.

We say that an SK-sequent $\Gamma \triangleright \Delta$ is G3SK-derivable (notation $\vdash^{\text {G3SK }} \Gamma \triangleright \Delta$, where the superscript will be omitted if clear from the context) iff there is a G3SK-derivation whose end-sequent is $\Gamma \triangleright \Delta$.

The calculus is sound and complete with respect to the semantic interpretation of SK-sequents, that is:

Theorem 1 (Soundness and completeness of G3SK, [12]) An SK-sequent $\Gamma \triangleright \Delta$ is G3SK-derivable iff it is valid.

Proof (sketch) The left-to-right direction (soundness) is easily established by checking for each rule that if the premises are satisfied by a valuation $v$ so is the conclusion (see [12] Proposition 3.3.4). Observe that the soundness of the negation rules is justified by the duality holding between strict and tolerant satisfaction (see Remark 2). 
The other direction (completeness) is established by showing that if the sequent $\Gamma \triangleright \Delta=\mathrm{s}: \Gamma_{1}, \mathrm{t}: \Gamma_{2} \triangleright \mathrm{s}: \Delta_{1}, \mathrm{t}: \Delta_{2}$ is not derivable, one can construct a countermodel for $\Gamma \triangleright \Delta$, that is, a valuation $v$ such that $v \vDash_{\mathrm{s}} A$ for all $A \in \Gamma_{1}, v \vDash_{\mathrm{t}} B$ for all $B \in \Gamma_{2}, v \not \nvdash_{\mathrm{s}} C$ for all $C \in \Delta_{1}$ and $v \not \nvdash_{\mathrm{t}} D$ for all $D \in \Delta_{2}$. One begins by applying the rules "backwards", i.e., bottom-up, starting from $\Gamma \triangleright \Delta$ until all formulas in all the leaves of the tree thereby obtained are labeled atoms. If all the leaves of the derivations are initial sequents of G3SK, the tree is an SK-derivation of the sequent. Otherwise, it contains at least one branch ending with an SK-sequent containing only labeled atoms which is however not an initial sequent of G3SK, say $\Phi \triangleright \Psi=\mathrm{s}: \Phi_{1}, \mathrm{t}: \Phi_{2} \triangleright \mathrm{s}: \Psi_{1}, \mathrm{t}: \Psi_{2}$. It is easy to establish that any function $v$ such that $v(P)=1$ for all $P \in \Phi_{1}, v(Q)=\frac{1}{2}$ for all $Q \in \Phi_{2}, v(R)=\frac{1}{2}$ for all $R \in \Psi_{1}$ and $v(Q)=0$ for all $Q \in \Psi_{2}$ is a valuation with the required properties (see, for a fully detailed proof, [12] Theorem 3.3.5).

Given Theorem 1 and Remark 6, the calculus G3SK allows to capture the four SK-notions of validity of an inference as follows:

Corollary $1 \vDash_{\mathrm{xy}} \Gamma \Rightarrow \Delta$ iff $\vdash^{\text {G3SK }} \mathrm{x}: \Gamma \triangleright \mathrm{y}: \Delta$

Example 2 To show that $\nvdash_{\mathrm{tt}} A \supset B, B \supset C \Rightarrow A \supset C$ (i.e., that the conditional of LP is not transitive) we extract a counter-model from a failed proof-search for the tt-sequent t: $P \supset Q, \mathrm{t}: Q \supset R \triangleright \mathrm{t}: P \supset R$. Backwards application of the rules of G3SK yields the following tree of SK-sequents:

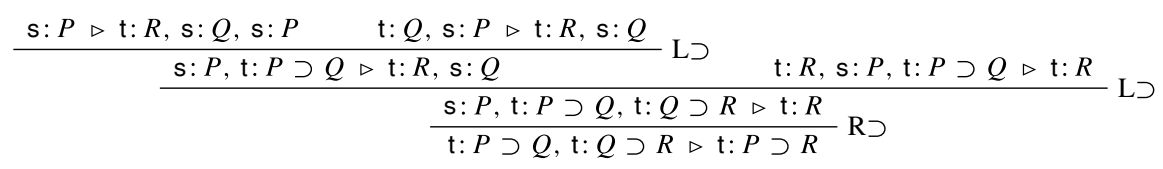

This is not a SK-derivation, since t: $Q, \mathrm{~s}: P \triangleright \mathrm{t}: R, \mathrm{~s}: Q$ is not an initial sequent. From this SK-sequent we extract a valuation $v$ assigning the values $1, \frac{1}{2}$ and 0 to $P$, $Q$ and $R$ respectively. The valuation tolerantly satisfies $P \supset Q$ and $Q \supset R$, but not $P \supset R$.

We conclude this section by observing that SK-sequents of the form $\mathrm{t}: P, \Phi \triangleright$ $\Psi$, s: $P$ cannot be taken as initial sequents, since they are unsound: when $P$ is only tolerantly satisfied, it may not be strictly satisfied. In the sequel, it will be however convenient to consider derivations with invalid initial sequents of this form. We call G3SK* the calculus obtained by extending G3SK with initial sequents of the form $\mathrm{t}: P, \Phi \triangleright \Psi, \mathrm{s}: P$. We will refer to these sequents as quasi-initial sequents, and to derivations in G3SK* as quasi-SK-derivations.

The failed proof-search in Example 2 yields a quasi-SK-derivation. This is however not always the case. For instance, by attempting to construct a derivation for the sequent t: $P \triangleright \mathrm{s}: Q \vee R$ one obtains a tree of sequents whose only branch ends with the sequent t: $P \triangleright \mathrm{s}: Q, \mathrm{~s}: R$, which is neither initial nor quasi-initial. 


\subsection{From G3C to G3SK and back via (un)labeling}

By removing all labels from the calculus G3SK we obtain (a minor variant of ${ }^{8}$ the standard calculus for classical logic G3C (see, e.g., [15]).

We call C-sequents all expressions of the form $\Gamma \triangleright \Delta$ in which $\Gamma$ and $\Delta$ are multisets of formulas $A \in \mathcal{L}$.

A G3C-derivation, or shortly a C-derivation, is a tree of C-sequents constructed according to the propositional rules obtained by removing all labels from those of Table 1 and whose leaves are C-sequents obtained by removing all labels from the initial sequents of G3SK.

We say that a $\mathrm{C}$-sequent $\Gamma \triangleright \Delta$ is G3C-derivable (notation $\vdash^{\mathrm{G} 3 \mathrm{C}} \Gamma \triangleright \Delta$ ), where the superscript will be omitted if clear from the context) iff there is a G3C-derivation whose end-sequent is $\Gamma \triangleright \Delta$.

The system G3C is sound and complete with respect to the classical interpretation of sequents in terms of $\mathrm{C}$-valuations:

Theorem $2 \vdash^{\mathrm{G} 3 \mathrm{C}} \Gamma \triangleright \Delta$ iff for all $C$-valuations $v$, if $v \vDash A$ for all $A \in \Gamma$, then $v \vDash B$ for some $B \in \Delta$.

We call unlabeling the operation of removing labels from SK-sequents and (quasi-) SK-derivations. If $\Gamma \triangleright \Delta$ is an SK-sequent, we indicate with $u(\Gamma \triangleright \Delta)$ its unlabeling, i.e., the $\mathrm{C}$-sequent obtained by removing all labels from $\Gamma \triangleright \Delta$. Analogously, if $\mathscr{D}$ is a quasi-SK-derivation, we indicate with $u(\mathscr{D})$ its unlabeling, i.e., the result of removing all labels from $\mathscr{D}$.

Remark 7 Clearly, not only the unlabeling of any SK-derivation, but also of any quasi-SK-derivations is a C-derivation.

We call labeling the operation of decorating each formula of a C-sequent and Cderivation with a label. In particular, we call any function $l$ mapping each formula occurrence in a C-sequent $\Gamma \triangleright \Delta$ onto $\{\mathrm{t}, \mathrm{s}\}$ a labeling function for $\Gamma \triangleright \Delta$, and we call the $l$-labeling of $\Gamma \triangleright \Delta$ (notation $l(\Gamma \triangleright \Delta))$ the SK-sequent obtained by replacing each formula occurrence $A$ in the $\mathrm{C}$-sequent $\Gamma \triangleright \Delta$ with the labeled formula $l(A): A$ (where $l(A)$ is the value of $l$ on the formula occurrence $A$ ).

When $l(\Gamma \triangleright \Delta)$ is an st-sequent (i.e., $l(\Gamma \triangleright \Delta)=\mathrm{s}: \Gamma \triangleright \mathrm{t}: \Delta)$, we say that $l(\Gamma \triangleright \Delta)$ is the st-labeling of $\Gamma \triangleright \Delta$, and we indicate it as $\operatorname{st}(\Gamma \triangleright \Delta)$. Similarly for tt, ss and ts.

Example 3 The tt-labeling of the C-sequent $P \supset Q, Q \supset R \triangleright P \supset R$ is the SK-sequent t: $P \supset Q, \mathrm{t}: Q \supset R \triangleright \mathrm{t}: P \supset R$

By reading the rules of G3SK backwards it is clear that the labels in the endsequent of a quasi-SK-derivation $\mathscr{D}$ uniquely determine the labels in all sequents in

\footnotetext{
${ }^{8}$ In usual formulations, one does not require atomic contexts $\Phi, \Psi$ in initial sequents. This however does not affect derivability, since initial sequents with arbitrary contexts are derivable from the restricted ones.
} 
$\mathscr{D}$. Thus, a quasi-SK-derivation $\mathscr{D}$ with end-sequent $\mathrm{s}: \Gamma_{1}, \mathrm{t}: \Gamma_{2} \triangleright \mathrm{s}: \Delta_{1}, \mathrm{t}: \Delta_{2}$ is uniquely determined by the C-derivation $u(\mathscr{D})$ obtained by unlabeling $\mathscr{D}$, together with the labeling function for the C-sequent $\Gamma_{1}, \Gamma_{2} \triangleright \Delta_{1}, \Delta_{2}$ that assigns $s$ to all elements of $\Gamma_{1}$ and $\Delta_{1}$ and to all elements of $\Gamma_{2}$ and $\Delta_{2}$.

Given a C-derivation $\mathscr{D}$ with end-sequent $\Gamma \triangleright \Delta$ and a labeling function $l$ for $\Gamma \triangleright \Delta$, we call the $l$-labeling of $\mathscr{D}$, notation $l(\mathscr{D})$, the quasi-SK-derivation that is uniquely determined by decorating each formula occurrence in $\mathscr{D}$ starting from the end-sequent in accordance with the rules of G3SK.

Observe that, in general, a labeling for the end-sequent may not induce an SKderivation, but only a quasi-SK-derivation. In case $l(\mathscr{D})$ is an SK-derivation we say that $l$ is a successful labeling of $\mathscr{D}$ or that $l(\mathscr{D})$ is successful.

The following example clarifies these definitions:

Example 4 Let $\mathscr{D}$ be the following C-derivation:

$$
\frac{P \triangleright R, Q, P \quad Q, P \triangleright R, Q}{\frac{P, P \supset Q \triangleright R, Q}{P} \mathrm{~L} \quad R, P, P \supset Q \triangleright R} \mathrm{~L} \supset
$$

The tt-labeling of $\mathscr{D}, \mathrm{tt}(\mathscr{D})$ is the quasi-SK-derivation of Example 2. Note that $\operatorname{tt}(\mathscr{D})$ is only a quasi-SK-derivation, and thus it is not a successful labeling of $\mathscr{D}$.

Although in general a labeling need not be successful, it is easy to see that the stlabeling of any C-derivation is successful, and that the ts-labeling of any C-derivation with a structural end-sequent is not successful:

Fact 1 For all C-derivations $\mathscr{D}, \operatorname{st}(\mathscr{D})$ is successful.

Proof The fact is established by observing that the end-sequent of the quasi-SKderivation $s t(\mathscr{D})$ is an st-sequent (i.e., it is of the form $s: \Gamma \triangleright t: \Delta$ ), and that if the end-sequent of a quasi-SK-derivation $\mathscr{D}$ is an st-sequent, then every sequent in $\mathscr{D}$ is an st-sequent, initial sequents included, and hence the quasi-SK-derivation is an SK-derivation as well.

Fact 1 shows that the labeling introduced to capture st-validities can therefore be seen as inducing an inessential (from a proof-theoretic point of view) decoration of a classical C-derivation, from which we can conclude the following:

Corollary 2 [6, 12] An inference is st-valid iff it is $C$-valid.

As we observed above in Section 2.2, the other SK-notions of validity are stricter than the notion of st- and hence of C-validity (i.e., the set of xy-valid inferences are all proper subsets of the set of $\mathrm{C}$-valid inference for $\mathrm{xy} \neq \mathrm{st}$.) Proof-theoretically, this is reflected by the fact that the labeling of a C-derivation according to the standards of a SK-notion of validity other than st-validity needs not be successful. The limit case is that of ts-validity, which is empty: 
Fact 2 There are no SK-derivable structural ts-sequents.

Proof Suppose by reductio that $\mathscr{D}$ is an SK-derivation of a ts-sequent. By inspecting the rules, it is clear that every sequent in $\mathscr{D}$ is a ts-sequent, including the leaves. If $\Gamma \triangleright \Delta$ is structural, no sequent in the leaves contains either $\perp$ or $T$ and thus none of them can be an initial sequent of G3SK (at best, a quasi-initial sequent), and hence $\mathscr{D}$ is not an SK-derivation.

Fact 2 immediately implies that:

\section{Corollary 3 [11] No structural inference is ts-valid.}

Dually, concerning unsatisfiability, we have that:

Fact 3 [22] The following holds:

1. An inference is ts-unsatisfiable iff it is C-unsatisfiable.

2. No structural inference is st-unsatisfiable. ${ }^{9}$

Proof Given that an inference $\Gamma \Rightarrow \Delta$ is xy-unsatisfiable iff all formulas in $\Gamma$ are $\mathrm{x}$-valid and all formulas in $\Delta$ are $\mathrm{y}$-unsatisfiable (see footnote 6 above), by completeness it follows that $(\Gamma \Rightarrow \Delta) \vDash_{\mathrm{xy}}$ iff for all $A \in \Gamma$ and $B \in \Delta$ all sequents $\triangleright \mathrm{x}: A$ and $y: B \triangleright$ are SK-derivable. If $x y=$ ts, by Fact 1 this is the case iff the corresponding sequents are $\mathrm{C}$-derivable, and hence iff the inference $\Gamma \Rightarrow \Delta$ is $\mathrm{C}$-unsatisfiable. If $x y=$ st and the inference $\Gamma \Rightarrow \Delta$ is structural, then by Fact 2 no such sequent is derivable.

\subsection{On Cut-elimination}

Given the interpretation of SK-sequents, the following form of cut is clearly sound:

$$
\frac{\Gamma \triangleright \Delta, \mathrm{x}: A \quad \mathrm{x}: A, \Gamma^{\prime} \triangleright \Delta^{\prime}}{\Gamma, \Gamma^{\prime} \triangleright \Delta, \Delta^{\prime}} C u t
$$

Moreover, the completeness of the cut-free calculus G3SK (Theorem 1) provides a (semantic) proof of its admissibility. ${ }^{10}$ Similarly for the other structural rules such as generalized identity, i.e., initial sequents without limitations to propositional variables (that we will employ in some examples below), weakening and contraction.

\footnotetext{
${ }^{9}$ Note that the "empty" inference $(\Rightarrow)$ is st-unsatisfiable, but it is not structural, since both its antecedent and succedent are empty.

${ }^{10} \mathrm{~A}$ rule is admissible iff (for all instances of the rule) whenever the sequents in the premises are derivable (i.e., valid) so is the conclusion sequent.
} 
Syntactic proofs of these results, as well as of the invertibility ${ }^{11}$ of all the rules of the system could be given by standard ${ }^{12}$ methods (see, e.g., [15] p. 53 ff.).

We observe moreover that the following form of cut

$$
\frac{\Gamma \triangleright \Delta, \mathrm{t}: A \quad \mathrm{~s}: A, \Gamma^{\prime} \triangleright \Delta^{\prime}}{\Gamma, \Gamma^{\prime} \triangleright \Delta, \Delta^{\prime}} C u t_{\mathrm{st}}
$$

is neither sound nor admissible with respect to the semantic interpretation of SKsequents, since it would allow one to infer from the valid sequents $\mathrm{t}: A \triangleright \mathrm{t}: A$ and $\mathrm{s}: A \triangleright \mathrm{s}: A$ the invalid sequent $\mathrm{t}: A \triangleright \mathrm{s}: A$. Not even the restriction of this form of cut to st-sequents is sound:

$$
\frac{\mathrm{s}: \Gamma \triangleright \mathrm{t}: \Delta, \mathrm{t}: A \quad \mathrm{~s}: A, \mathrm{~s}: \Gamma^{\prime} \triangleright \mathrm{t}: \Delta^{\prime}}{\mathrm{s}: \Gamma, \mathrm{s}: \Gamma^{\prime} \triangleright \mathrm{t}: \Delta, \mathrm{t}: \Delta^{\prime}}
$$

which is the way in which the unsoundness of Cut for ST is reflected in G3SK. ${ }^{13}$

Remark 8 All the mentioned results, in particular completeness and admissibility of structural rules could also be established for a first-order version of the calculus. In this case, however, to obtain the admissibility of contraction the principal formula in the left rule for the universal quantifer, $\forall \mathrm{L}$, and in the right rule for the existential quantifier, $\exists \mathrm{R}$, have to be repeated in the premises, e.g.,:

$$
\frac{\mathrm{x}: A(t), \mathrm{x}: \forall x \cdot A, \Gamma \triangleright \Delta}{\mathrm{x}: \forall x \cdot A, \Gamma \triangleright \Delta}
$$

This induces some (minor) complication in the completeness proof (in particular, termination is no more obvious, nor can initial sequents be restricted to atoms as we did in G3SK). In the case of G3SK however no repetition of this kind is needed (like in the calculus for classical propositional logic) and hence the simplified proof of completeness sketched above suffices, and the admissibility of structural rules follows literally the one given by [15, p. $53 \mathrm{ff}$.] (since the rules of G3SK are just a decoration of the G3 rules for propositional classical logic, with labels playing no particular role in the proofs of admissibility). We also remark that Girard's extended his system (as well as the proof of completeness) to a second-order language.

\footnotetext{
${ }^{11} \mathrm{~A}$ rule is invertible iff whenever the sequent in the conclusion is derivable so are the sequents in the premises.

${ }^{12}$ One of the referees objected that the methods employed by Negri and von Plato are not standard, in that they rely on a more subtle analysis of derivations than the methods underlying Gentzen's original proof of cut elimination. It is certainly true that what deserves the label standard is (to some extent at least) a subjective issue. It is however also true that the methods referred to are by now widely used. See also Section 10 for further discussion.

${ }^{13}$ Note that although the rule is unsound, it is admissible as a consequence of the fact that the unlabeling of an SK-derivation is a C-derivation, of the admissibility of cut in G3C and of Fact 1. However, the rule ceases to be admissible when the language of G3SK is extended for instance with a formula, say $*$, which takes value $\frac{1}{2}$ in all interpretations. In the extension G3SK to this language both $\triangleright \mathrm{t}: *$ and $\mathrm{s}: * \triangleright$ are valid, but not the empty sequent.
} 


\section{From Inferences to Higher-level Inferences}

Following [3], we generalize the notion of inference discussed in the previous sections by introducing for each natural number $n$ the notion of inference of level $n$. In this hierarchy of notions, the inferences discussed in the previous sections play the role of inferences of level 1 , and formulas can be seen as the "degenerate case" of inferences, i.e., inferences of level 0 . The idea at the basis of this generalization of the notion of inference can be made precise by defining the notion of inference of level $n$, short $n$-inference, as follows:

Definition 1 By induction on $n .^{14}$

- For all $A \in \mathcal{L}, A$ is a 0-inference;

- If $\Gamma$ and $\Delta$ are finite multisets of $n$-inferences, then $(\Gamma \Rightarrow \Delta)$ is a $n+1$-inference.

Remark 9 We will use capital Greek letters not just for multisets of formulas, but for multisets of inferences as well. Below we will use them also for multisets of labeled inferences (to be introduced below). As we will stress, the context will always make clear what kind of multiset is meant, and thus with a little care, no confusion should arise for the reader. Moreover, for ease of presentation it will be convenient to use capital roman letters $A, B, C$ not only for formulas but also for inferences of any level.

Remark 10 To increase readability, we will sometimes use $\Rightarrow$ instead of $\Rightarrow$ for the (main) arrow of an inference of level 2.

Example 5 We thus have that:

(i) $A \supset B, B \supset C \Rightarrow A \supset C$ is an inference of level 1

(ii) $(A \Rightarrow B),(B \Rightarrow C) \Rightarrow(A \Rightarrow C)$ is an inference of level 2.

Remark 11 Inferences of level 2 are often referred to as "meta-inferences", and some

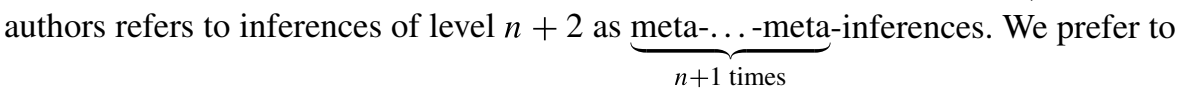
talk about inferences of different levels since, besides allowing for a more straightforward definition of the hierarchy of notions that includes both formulas and inferences as limit cases, it also avoids the impression that a semantic ascent from the object language to the meta-language is taking place (an inference of any level is an object language entity as much as a formula). Object language entities of different levels are well-known in proof theory (see, e.g., Schroeder-Heister's higher-level rules [24], or Kosta Došen's higher-level sequents [10]).

\footnotetext{
${ }^{14}$ Here and below, the final clauses of inductive definitions, e.g., "Nothing else is an $n$-inference." will be omitted.
} 


\section{Higher-level Inferences, Classically}

A natural way of scaling the notion of C-validity to inferences of level 2 is by saying that a 2 -inference $\Gamma \Rightarrow \Delta$ is $\mathrm{C}$-satisfied by a C-valuation $v$ iff $v$ does not C-satisfies a 1-inference $\Sigma \Rightarrow \Theta \in \Gamma$ or it C-satisfies a 1-inference $\Sigma^{\prime} \Rightarrow \Theta^{\prime} \in \Delta$. A 2-inference is $\mathrm{C}$-valid, when it is $\mathrm{C}$-satisfied by every $\mathrm{C}$-valuation, and $\mathrm{C}$-unsatisfiable when it is C-satisfied by no valuation. ${ }^{15}$

Clearly, the same definition can be lifted to inferences of any level. A bit more formally, one can define by induction on $n$ a hierarchy of notions of satisfaction (we indicate each of them as C-satisfaction ${ }^{n}$ for some $n \in \mathbb{N}$, notation $\models^{n}$ ): the base of the hierarchy is the notion of $\mathrm{C}$-satisfaction ${ }^{0}$ being just the definition of $\mathrm{C}$-satisfaction for formulas of Section 2.1 above; the notion of $\mathrm{C}$-satisfaction ${ }^{n+1}$ for inferences of level $n+1$ is defined in terms of the notion of C-satisfaction ${ }^{n}$ for inferences of level $n$ as follows:

$$
v \vDash^{(n+1)}(\Gamma \Rightarrow \Delta) \text { iff if } v \vDash^{n} A \text { for all } A \in \Gamma \text { then } v \vDash^{n} B \text { for some } B \in \Delta
$$

The generalization of the standard notion of inference by allowing inferences of higher-levels does not seem to enrich the classical setting in a substantial way. To clarify the sense in which in the classical setting higher-level inferences can be reduced to inferences of level 1 , and in turn to those of level 0 , we recall the idea of "lowering of an inference" i.e., of mapping an inference of level $n \geq 1$ onto an inference of level $n-1:^{16}$

Definition 2 The lowering of an inference $A_{1}, \ldots, A_{p} \Rightarrow B_{1}, \ldots, B_{q}$ of level $m=1+n$, indicated as $\operatorname{low}\left(A_{1}, \ldots, A_{p} \Rightarrow B_{1}, \ldots, B_{q}\right)$ is defined by induction on $n$ as follows:

- If $n=0$, then if $p>0$ and $q>0$ every $A_{i}$ and $B_{j}$ (for $1 \leq i \leq p$ and $1 \leq j \leq q$ ) are formulas, and we define

$$
\operatorname{low}\left(A_{1}, \ldots, A_{p} \Rightarrow B_{1}, \ldots, B_{q}\right)=\bigwedge_{i=1}^{p} A_{i} \supset \bigvee_{j=1}^{q} B_{j}
$$

(where $\bigwedge_{i=1}^{p} A_{i}=\left(A_{1} \wedge\left(\ldots \wedge A_{p}\right) \ldots\right.$ ) if $p \geq 1$, and $\bigwedge_{i=1}^{p} A_{i}=\top$ otherwise; and $\bigvee_{j=1}^{q} B_{j}=\left(B_{1} \vee\left(\ldots \vee B_{q}\right) \ldots\right)$ if $q \geq 1$, and $\bigvee_{j=1}^{q} B_{j}=\perp$ otherwise $)$

- If $n>0$, then

$$
\operatorname{low}\left(A_{1}, \ldots, A_{p} \Rightarrow B_{1}, \ldots, B_{q}\right)=\operatorname{low}\left(A_{1}\right), \ldots, \operatorname{low}\left(A_{p}\right) \Rightarrow \operatorname{low}\left(B_{1}\right), \ldots, \operatorname{low}\left(B_{q}\right)
$$

\footnotetext{
${ }^{15}$ These definitions and those of the following section are adapted from [9] and are also used in [3]. The idea of validity for a meta-inference is also present in [2], although in a slightly different ("global") sense. ${ }^{16}$ This terminology was introduced by Dicher and Paoli for inferences of level 1, and the notion has been generalized by Barrio et al. to (single-conclusion) inferences of higher level. Here we generalize it to multiple-conclusions higher-level inferences in the obvious way.
} 
Moreover, the $n$-lowering of an inference $A$ of level $m \geq n$, to be indicated with $\operatorname{low}^{n}(A)$ is defined by induction on $n$ as follows (note that if $A$ is of level $m<n$ the $n$-lowering of $A$ is undefined):

- $\operatorname{low}^{0}(A)=A$

- $\operatorname{low}^{n+1}(A)=\operatorname{low}\left(\operatorname{low}^{n}(A)\right)$

Clearly, if $A$ is an $n$-inference and $n \geq m, \operatorname{low}^{m}(A)$ is an $(n-m)$-inference (and if $n \geq 1$ then $\operatorname{low}(A)$ is an $(n-1)$-inference).

The following holds:

Fact 4 For all $n$-inferences $\Gamma \Rightarrow \Delta, v \vDash^{n} \Gamma \Rightarrow \Delta$ iff $v \vDash^{0} \operatorname{low}^{n}(\Gamma \Rightarrow \Delta)$

That is, in the classical setting the notion of validity for inferences of higher-level can always be reduced to those of level 0 , and thus inferential arrows of any level are just a notational variant of the conditional. As we will see later, this is not the case in the SK-setting, and thus the addition of inferences of higher-level is a substantial enrichment of that setting.

Before turning to inferences of higher-level in the SK-setting, we wish to briefly show how the classical sequent calculus G3C discussed in the previous section can be generalized to a calculus $\mathrm{G} 3 \mathrm{C}^{\omega}$ capable of capturing not only the validity of 1 -inferences, but of inferences of any arbitrary level. Although in the light of the previous result this extension is not very interesting per se, we believe that it will help the reader in better understanding the functioning of the analogous generalization of G3SK that we will introduce later on.

The basic idea yielding the calculus $\mathrm{G}_{3} \mathrm{C}^{\omega}$ is that of allowing not only formulas, but also inferences of arbitrary level to occur to the left and to the right of the sequent symbol. Let us first describe the form and the semantic interpretation of the sequents of the fragment $\mathrm{G}_{3} \mathrm{C}^{1}$ of $\mathrm{G} 3 \mathrm{C}^{\omega}$ in which we allow only inferences of level $\leq 1$ to occur within sequents.

We call a $C^{1}$-sequent any expression of the form $\Gamma \triangleright \Delta$, where $\Gamma$ and $\Delta$ are finite multisets of inferences of level $\leq 1$. In general a $C^{1}$-sequent can be depicted as:

$$
\Gamma_{0}, \Gamma_{1} \triangleright \Delta_{0}, \Delta_{1}
$$

where $\Gamma_{0}$ and $\Delta_{0}$ are (possibly empty) finite multisets of formulas (i.e., of 0 -inferences) and $\Gamma_{1}$ and $\Delta_{1}$ are (possibly empty) multisets of 1-inferences. Semantically, we say that such a sequent is satisfied in a C-valuation $v 0$ - iff if all formulas in $\Gamma_{0}$ are $\mathrm{C}$-satisfied ${ }^{0}$ by $v$ and all inferences in $\Gamma_{1}$ are C-satisfied ${ }^{1}$ by $v$, then either at least one of the formulas in $\Delta_{0}$ is C-satisfied ${ }^{0}$ by $v$ or at least one of the inferences in $\Delta_{1}$ are $\mathrm{C}$-satisfied ${ }^{1}$ by $v$. A sequent is valid iff it is satisfied in all $\mathrm{C}$-valuations.

Remark 12 From the notion of satisfaction (resp. validity) of a $\mathrm{C}^{1}$-sequent, we can recover both that of C-satisfaction ${ }^{1}\left(\mathrm{C}\right.$-validity ${ }^{1}$ ) of a 1-inference (when $\Gamma_{1}$ and $\Delta_{1}$ are empty, a sequent of the above is satisfied by $v$ iff the 1 -inference $\Gamma_{0} \Rightarrow \Delta_{0}$ is Csatisfied $^{1}$ by $v$ ), as well as that of C-satisfaction ${ }^{2}\left(\mathrm{C}\right.$-validity $\left.{ }^{2}\right)$ of a 2-inference (when 
$\Gamma_{0}$ and $\Delta_{0}$ are both empty, a sequent of the above form is satisfied by $v$ iff so the 2inference $\Gamma_{1} \Rightarrow \Delta_{1}$ is C-satisfied ${ }^{2}$ by $v$ ). Similarly for the corresponding notions of unsatisfiability.

Note that the $C^{1}$-satisfaction/validity (resp. non-satisfaction/unsatisfiability) of a 1-inference $\Gamma \Rightarrow \Delta$ are equivalent to the satisfaction/validity of the $C^{1}$-sequent $\triangleright(\Gamma \Rightarrow \Delta)(\operatorname{resp} .(\Gamma \Rightarrow \Delta) \triangleright)$.

In the case of the full calculus $\mathrm{G}_{3} \mathrm{C}^{\omega}$, a $\mathrm{C}^{\omega}$-sequent is any expression of the form $\Gamma \triangleright \Delta$, where $\Gamma$ and $\Delta$ are finite multisets of inferences of any level. In general a $\mathrm{C}^{\omega}$-sequent can be depicted as:

$$
\Gamma_{0}, \Gamma_{1}, \Gamma_{2}, \ldots \triangleright \Delta_{0}, \Delta_{1}, \Delta_{2}, \ldots
$$

where $\Gamma_{n}$ and $\Delta_{n}$ are (possibly empty) finite multisets of $n$-inferences for all $n \in \mathbb{N}$. ${ }^{17}$

Remark 13 The notion of satisfaction for $\mathrm{C}^{\omega}$-sequents is the obvious generalization of that for $\mathrm{C}^{1}$-sequents, and Remark 12 also scales to higher-level inferences.

The sequent calculus $\mathrm{G}_{3} \mathrm{C}^{\omega}$ is obtained from the sequent calculus $\mathrm{G} 3 \mathrm{C}$ by adding a left and right rule to introduce inferences of level $\geq 1$ in the succedent of a sequent:

$$
\frac{\Gamma, \Theta \triangleright \Sigma, \Delta}{\Theta \triangleright \Sigma,(\Gamma \Rightarrow \Delta)} \mathrm{R} \Rightarrow
$$

and in the antecedent of a sequent (the rule has $n+m$ premises):

$$
\frac{\Theta \triangleright \Sigma, A_{1} \quad \ldots \quad \Theta \triangleright \Sigma, A_{n} \quad B_{1}, \Theta \triangleright \Sigma \quad \ldots \quad B_{m}, \Theta \triangleright \Sigma}{\left(A_{1}, \ldots, A_{n} \Rightarrow B_{1}, \ldots, B_{m}\right), \Theta \triangleright \Sigma} \mathrm{L} \Rightarrow
$$

(Clearly, applications of the right rule are subject to the restriction that both $\Gamma$ and $\Delta$ can contain only inferences of a given level $n$, otherwise the conclusion of the rule would not be a well formed sequent; similarly, in applications of the left rule all $A_{i}$ and $B_{j}$ (for $1 \leq i \leq n$ and $1 \leq j \leq m$ ) must all be inferences of the same level. Observe also that the Greek letters in these rules, as well as in those of G3C now stand for multisets containing inferences of any level.)

The notion of derivability in $\mathrm{G} 3 \mathrm{C}^{\omega}$, which we indicate with $\vdash^{\mathrm{G} 3 \mathrm{C}^{\omega}}$, captures that of validity of $\mathrm{C}^{\omega}$-sequents in the following way:

Theorem 3 A $\mathrm{C}^{\omega}$-sequent is $\mathrm{G} \mathrm{C}^{\omega}$-derivable iff it is valid.

Proof The proof of this statement is a straightforward generalization of the proof of completeness for $\mathrm{G} 3 \mathrm{C}$.

Corollary 4 For any $n$-inference $\Gamma \Rightarrow \Delta$ :

$$
\vDash^{n}(\Gamma \Rightarrow \Delta) \text { iff } \vdash^{\mathrm{G}^{3} \mathrm{C}^{\omega}} \Gamma \triangleright \Delta \text { iff } \vdash^{\mathrm{G} 3 \mathrm{C}^{\omega}} \triangleright(\Gamma \Rightarrow \Delta)
$$

\footnotetext{
${ }^{17}$ Note that since the antecedent and the succedent are finite multisets, only finitely many among the $\Gamma_{n}$ and $\Delta_{n}$ are non-empty.
} 
Corollary 5 For any $n$-inference $\Gamma \Rightarrow \Delta$ :

$$
(\Gamma \Rightarrow \Delta) \vDash^{n} \text { iff } \vdash^{\mathrm{G}^{3} \mathrm{C}^{\omega}}(\Gamma \Rightarrow \Delta) \triangleright
$$

Proof The corollaries follows from Remarks 12 and 13.

Remark 14 Similarly to what we have already seen for G3C, it is easy to show that the calculus syntactically admits structural rules such as generalized identity and cut as well as invertibility of the $\mathrm{L} \Rightarrow$ and $\mathrm{R} \Rightarrow$ rules.

\section{Higher-level Inferences in the SK-setting}

In the SK-setting, a "simple-minded" way of defining SK-notions of satisfaction for inferences of level 2 is by saying that a 2 -inference $\Gamma \Rightarrow \Delta$ is xy-satisfied by a valuation $v$ iff if $v$ xy-satisfies all inferences $\Sigma \Rightarrow \Theta \in \Gamma$, then it xy-satisfies at least one inference $\Sigma^{\prime} \Rightarrow \Theta^{\prime} \in \Delta$, and the xy-validity/unsatisfiability of a 2-inference can be defined as usual by quantifying over all valuations.

Remark 15 The notions of validity resulting by these four simple-minded notions of satisfaction of inferences of level 2 are commonly referred to in the literature as K3-, LP- ST- and TS-validity of metainferences.

As first observed by [3], one can define more SK-notions of satisfaction for 2inferences by setting different standards of satisfaction on the 1-inferences in the antecedent and in the consequent (in the same way in which one can consider, besides ss- and tt-satisfaction, also st- and ts-satisfaction for 1-inferences). For instance, we may say that a valuation $v$ stts-satisfies a 2 -inference $\Gamma \Rightarrow \Delta$ iff if all the inference in $\Gamma$ are st-satisfied by $v$ then at least one inference in $\Delta$ is ts-satisfied by $v$. In general, we will define the notion of a 2 -inference $\Gamma \Rightarrow \Delta$ being xywz-satisfied by a valuation $v$, written $v \vDash_{\text {xywz }} \Gamma \Rightarrow \Delta$, as follows:

$$
\begin{array}{r}
v \vDash_{\mathrm{xywz}} \Gamma \Rightarrow \Delta \text { iff if } v \vDash_{\mathrm{xy}} \Sigma \Rightarrow \Theta(\text { for all } \Sigma \Rightarrow \Theta \in \Gamma \text { ) } \\
\text { then } v \vDash_{\mathrm{wz}} \Sigma^{\prime} \Rightarrow \Theta^{\prime}\left(\text { for some } \Sigma^{\prime} \Rightarrow \Theta^{\prime} \in \Delta\right. \text { ) }
\end{array}
$$

The four simple-minded notions of xy-satisfaction of 2-inferences are hence just limit cases of this more general notion in which $\mathrm{x}=\mathrm{w}$ and $\mathrm{y}=\mathrm{z}$.

A quick count shows that we have in general 16 different notions of SKsatisfaction (and hence of validity and unsatisfiability) for 2-inferences. As the different standards of the SK-notions of satisfaction of formulas induces a partial order on the SK-notions of satisfaction of 1-inferences (see above Fig. 1), the latter order induces one among the 16 SK-notions of satisfaction for 2-inferences, the Hasse diagram of which is depicted in Fig. 2. As before, an SK-notion of satisfaction is more tolerant than another iff it requires either more tolerant standards of satisfaction on the succedent or stricter standards on the antecedent (for an investigation of the structural properties of these notions of satisfaction, see [16]). 


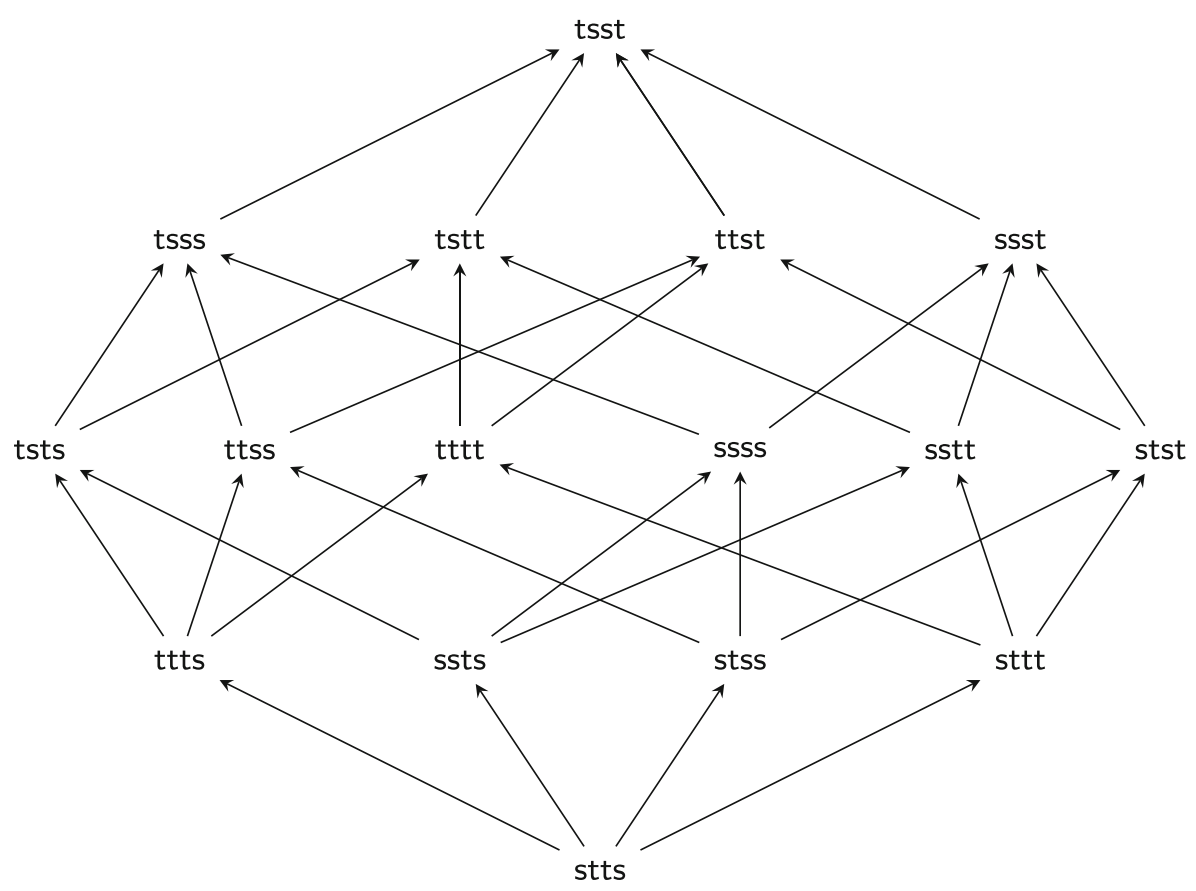

Fig. 2 Relationships between the notions of satisfaction for 2-inferences

As [22] observed, these considerations scale straightforwardly to inferences of any level (for analogous definitions see also [16, 17, 23] the latter for a generalization into the transfinite). Observe that we have two notions of satisfaction-tolerant and strict-for formulas, (i.e., 0-inferences); for 1-inferences (of level 1) we have four $\left(2^{2}\right)$ and for 2-inferences we have $16\left(2^{2^{2}}\right)$ notions of satisfaction. In general, for inferences of level $n$ we can define $\underbrace{2^{2}}_{n+1 \text {-times }}$ notions of satisfaction (and hence of validity and unsatisfiability). Each such notion is identified by a pair of labels identifying the notions of satisfaction to be applied to the inferences in the antecedent and in the succedent. We thus define the notion of label of level $n$, short $n$-label, as follows:

Definition 3 By induction on $n$.

- $\quad t$ and $s$ are the only 0-labels;

- If I and I' are $n$-labels then $\mathrm{II}^{\prime}$ is a $n+1$-label.

For every label $\mathrm{II}^{\prime}$ of level $n \geq 1$ we have a corresponding SK-notion of satisfaction for $n$-inferences defined in terms of the two notions of satisfaction for $(n-1)$-inferences corresponding to the $(n-1)$-labels I and I':

$$
v \vDash_{\mathbb{I}^{\prime}} \Gamma \Rightarrow \Delta \text { iff if } v \vDash_{\mathrm{I}} A \text { (for all } A \in \Gamma \text { ) then } v \vDash_{\mathbb{I}^{\prime}} B \text { (for some } B \in \Delta \text { ) }
$$


The ordering between strict and tolerant satisfaction of formulas induces an ordering on the notions of satisfaction, validity and unsatisfiability for $n$-inferences for any level $n$. Formally, we define a partial ordering $<$ on labels, so that $\mathrm{I}<\mathrm{I}^{\prime}$ expresses the fact that $I^{\prime}$-satisfaction is more tolerant than I-satisfaction, i.e., that if an inference of the appropriate level is I-satisfied by a valuation $v$ then it is I'-satisfied by $v$ as well and hence that I-validity entails $\mathrm{I}^{\prime}$-validity and $\mathrm{I}^{\prime}$-unsatisfiability entails I-unsatisfiability.

Definition 4 The relation $<$ is the transitive closure of the relation $\prec$ that we define by induction on $n$ as follows:

- $s \prec t$

- $\left(I_{1} I_{2}\right) \prec\left(I_{1}^{\prime} I_{2}^{\prime}\right)$ iff

- either $I_{1}=I_{1}^{\prime}$ and $I_{2} \prec I_{2}^{\prime}$;

- or $\mathrm{I}_{2}=\mathrm{I}_{2}^{\prime}$ and $\mathrm{I}_{1}^{\prime} \prec \mathrm{I}_{1}$.

In Section 9 we will give a proof that all these notions of validity and unsatisfiability (and hence of satisfaction as well) are pairwise distinct.

\section{The System G3SK ${ }^{\omega}$}

In order to capture the SK-notions of validity for higher-level inferences in a prooftheoretic fashion, in this section we introduce an extension of Girard's system G3SK, to be called $\mathrm{G}_{3} \mathrm{SK}^{\omega}$, in a way analogous to the one in which we extended G3C to $\mathrm{G}_{3} \mathrm{C}^{\omega}$ in the previous section. As formulas occur in SK-sequents accompanied by a label, so do $n$-inferences occur in the sequents of $\mathrm{GSSK}^{\omega}$ together with an $n$-label. For $n \geq 1$, a labeled $n$-inference is an expression of the form I: $\Gamma \Rightarrow \Delta$, where $\Gamma \Rightarrow \Delta$ is an $n$-inference and I is an $n$-label. To save space, we will often write a labeled inference of level $n \geq 1 \mathrm{I}: \Gamma \Rightarrow \Delta$ as $\Gamma \stackrel{\mathrm{l}}{\Rightarrow} \Delta .{ }^{18}$ When $\Gamma$ is a multiset of inferences, we indicate with I: $\Gamma$ the multiset of labeled inferences $\{\Sigma \stackrel{\text { l }}{\Rightarrow} \Theta \mid \Sigma \Rightarrow \Theta \in \Gamma\}$. An SK $^{\omega}$-sequent is an expression of the form $\Gamma \triangleright \Delta$, where $\Gamma$ and $\Delta$ are finite multisets containing labeled inferences of any level.

As we did for $\mathrm{G}_{3} \mathrm{C}^{\omega}$, in order to get familiar with $\mathrm{G}_{3} \mathrm{SK}^{\omega}$ we first briefly discuss its fragment $\mathrm{G}_{3} \mathrm{SK}^{1}$ in which only labeled inferences of level $\leq 1$ are allowed to occur in sequents. The general form of an $\mathrm{SK}^{1}$-sequent is thus the following (we separate the sequent into its antecedent and succedent, which are displayed in separate lines):

$$
\begin{aligned}
\mathrm{t}: \Gamma_{1}, \mathrm{~s}: \Gamma_{2}, \mathrm{tt}: \Sigma_{1}, \text { ts: } \Sigma_{2}, \text { st: } \Sigma_{3}, \text { ss }: \Sigma_{4} \triangleright \\
\quad \triangleright t: \Delta_{1}, \mathrm{~s}: \Delta_{2}, \mathrm{tt}: \Theta_{1}, \text { ts }: \Theta_{2}, \text { st }: \Theta_{3}, \text { ss }: \Theta_{4}
\end{aligned}
$$

where $\Gamma_{i}$ and $\Delta_{i}$ are (possibly empty) finite multisets of formulas (for $i=1,2$ ) and $\Sigma_{j}$ and $\Theta_{j}$ are multisets of inferences of level 1 (for $j=1,2,3,4$ ). We say that a

\footnotetext{
${ }^{18}$ To avoid confusion, let us stress that in a labeled $(n+1$-)inference $\Gamma \stackrel{\perp}{\Rightarrow} \Delta$, only the main inference arrow carries a label whereas the $n$-inferences in $\Gamma$ and $\Delta$ are unlabeled.
} 
$\mathrm{SK}^{1}$-sequent is satisfied by $v$ iff if all the formulas in $\Gamma_{1}$ are tolerantly satisfied by $v$, $\ldots$ and all the inferences in $\Sigma_{1}$ are tt-satisfied by $v, \ldots$ then either one of the formulas in $\Delta_{1}$ is tolerantly satisfied by $v \ldots$ or one of the inferences in $\Theta_{1}$ is tt-satisfied by $v$ .... Validity is defined as usual.

Remark 16 As for the previously discussed notions of satisfaction, validity and unsatisfiability of a sequent, from the notion of satisfaction (resp. validity and unsatisfiability) of a SK ${ }^{1}$-sequent we can recover all SK-notions of satisfaction (validity) of 1-inferences and of 2-inferences. For instance, when in a sequent of the above form all multisets of labeled inferences but $\Gamma_{1}$ and $\Delta_{2}$ are empty, we have a sequent of the form $\mathrm{t}: \Gamma_{1} \triangleright \mathrm{s}: \Delta_{2}$ that is satisfied by $v$ iff the 1-inference $\Gamma_{1} \Rightarrow \Delta_{2}$ is ts-satisfied by $v$; or when all multisets but $\Sigma_{4}$ and $\Theta_{2}$ are empty, we have a sequent of the form ss: $\Sigma_{4} \triangleright$ ts: $\Theta_{2}$ that is satisfied by $v$ iff the 2-inference $\Sigma_{4} \Rightarrow \Theta_{2}$ is ssts-satisfied by $v$.

Note that the $x y$-satisfaction/xy-validity (respectively non-xy-satisfaction/ $x y$ unsatisfiability) of a 1-inference $\Gamma \Rightarrow \Delta$ are equivalent to the $x y$-satisfaction/ validity of the $\mathrm{SK}^{1}$-sequent $\triangleright(\Gamma \stackrel{\text { xy }}{\Rightarrow} \Delta)($ resp. $(\Gamma \stackrel{\text { xy }}{\Rightarrow} \Delta) \triangleright)$.

The notion of satisfaction of an $\mathrm{SK}^{\omega}$-sequent is obtained by appropriately scaling that of satisfaction of an $\mathrm{SK}^{1}$-sequent. Remark 16 scales accordingly to all SK-notions of satisfaction and validity of inferences of any level.

Remark 17 To stress the connection between the satisfaction of an $\mathrm{SK}^{\omega}$-sequent of the form I: $\Gamma \triangleright \mathrm{I}^{\prime}: \Delta$ (where I and $\mathrm{I}^{\prime}$ are labels of the same level $n$ ) and the $\mathrm{II}^{\prime}$ satisfaction of $\Gamma \Rightarrow \Delta$ we will refer to a sequent of this form as an $I^{\prime}$-sequent.

The system G3SK ${ }^{\omega}$ is obtained by adding to G3SK rules to introduce labeled inferences of level $n$ to the right of the sequent symbol (here $\Gamma$ and $\Delta$ must both consist of inferences of the same level $n-1$ ):

$$
\frac{\mathrm{I}: \Gamma, \Theta \triangleright \Sigma, \mathrm{I}^{\prime}: \Delta}{\Theta \triangleright \Sigma,\left(\Gamma \stackrel{\mathrm{I}^{\prime}}{\Rightarrow} \Delta\right)} \mathrm{R} \Rightarrow
$$

and to the left of the sequent symbol (the rule has $p+q$ premises, and all $A_{i} \mathrm{~s}$ and $B_{j}$ s must be inferences of the same level $\left.n-1\right)$ :

$$
\frac{\Theta \triangleright \Sigma, \mathrm{I}: A_{1} \quad \ldots \quad \Theta \triangleright \Sigma, \mathrm{I}: A_{p} \quad \mathrm{I}^{\prime}: B_{1}, \Theta \triangleright \Sigma \quad \ldots \quad \mathrm{I}^{\prime}: B_{q}, \Theta \triangleright \Sigma}{\left(A_{1}, \ldots, A_{p} \stackrel{\mathrm{I}^{\prime}}{\Rightarrow} B_{1}, \ldots, B_{q}\right), \Theta \triangleright \Sigma} \mathrm{L} \Rightarrow
$$

The system G3SK ${ }^{\omega}$ is sound and complete with respect to the notion of validity of $\mathrm{SK}^{\omega}$-sequents:

Theorem 4 An SK ${ }^{\omega}$-sequent is derivable iff it is valid.

The proof of this statement is a straightforward extension of that of Theorem 3: from a failed proof-search for a sequent we can extract a counter-model. 
Corollary 6 For any $n$-inference $\Gamma \Rightarrow \Delta$ :

$$
\vDash_{\mathbb{I}^{\prime}} \Gamma \Rightarrow \Delta \text { iff } \vdash^{\mathrm{G} 3 \mathrm{GK}^{\omega}} \mathrm{I}: \Gamma \triangleright \mathrm{I}^{\prime}: \Delta \text { iff } \vdash^{\mathrm{G}^{\mathrm{GSK}} \mathrm{K}^{\omega}} \triangleright\left(\Gamma \stackrel{\mathrm{I}^{\prime}}{\Rightarrow} \Delta\right)
$$

Corollary 7 For any $n$-inference $\Gamma \Rightarrow \Delta$ :

$$
(\Gamma \Rightarrow \Delta) \vDash_{I^{\prime}} \quad \text { iff } \vdash^{\mathrm{G}^{\prime} \mathrm{SK}^{\omega}}\left(\Gamma \stackrel{\mathrm{I}^{\prime}}{\Rightarrow} \Delta\right) \triangleright
$$

We conclude this section with a short remark on the admissibility of structural rules and three examples which we will discuss in the next section.

Remark 18 As for $\mathrm{G} \mathrm{C}^{\omega}$, the calculus syntactically admits structural rules such as generalized identity (to be used in the examples below) and a schema for cut at different inference levels, as well as the invertibility of all rules. Again, the admissibility of these forms of Cut does not contradict the fact that some $\mathrm{SK}^{\omega}$-notions of satisfaction may not be closed under transitivity.

$$
\frac{\Gamma \triangleright \Delta, \mathrm{I}: A \quad \mathrm{I}: A, \Gamma^{\prime} \triangleright \Delta^{\prime}}{\Gamma, \Gamma^{\prime} \triangleright \Delta, \Delta^{\prime}} C u t
$$

Example $6 \vDash_{\mathrm{tttt}} A \Rightarrow B, B \Rightarrow C \Rightarrow A \Rightarrow C$

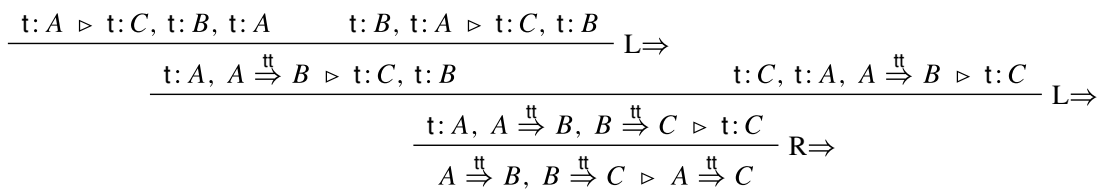

Example $7 \vDash_{\text {tsst }} A \Rightarrow B, B \Rightarrow C \Rightarrow A \Rightarrow C$.

$$
\begin{gathered}
\frac{\mathrm{s}: A \triangleright \mathrm{t}: C, \mathrm{t}: B, \mathrm{t}: A \quad \mathrm{~s}: B, \mathrm{~s}: A \triangleright \mathrm{t}: C, \mathrm{t}: B}{\mathrm{~s}: A, A \stackrel{\mathrm{ts}}{\Rightarrow} B \triangleright \mathrm{t}: C, \mathrm{t}: B} \mathrm{~L} \Rightarrow \quad \mathrm{s}: C, \mathrm{~s}: A, A \stackrel{\mathrm{ts}}{\Rightarrow} B \triangleright \mathrm{t}: C \\
\frac{\mathrm{s}: A, A \stackrel{\mathrm{ts}}{\Rightarrow} B, B \stackrel{\mathrm{ts}}{\Rightarrow} C \triangleright \mathrm{t}: C}{A \stackrel{\mathrm{ts}}{\Rightarrow} B, B \stackrel{\mathrm{ts}}{\Rightarrow} C \triangleright A \stackrel{\text { st }}{\Rightarrow} C} \mathrm{R} \Rightarrow
\end{gathered}
$$

Example $8 \not \nvdash_{\text {stst }} P \Rightarrow Q, Q \Rightarrow R \Rightarrow P \Rightarrow R$, i.e., transitivity for ST-inferences is not valid.

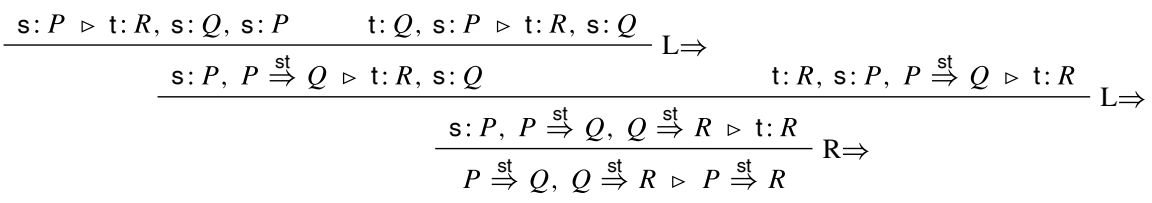

From the quasi-initial sequent $\mathrm{t}: Q, \mathrm{~s}: P \triangleright \mathrm{t}: R, \mathrm{~s}: Q$ we construct a valuation assigning 1 to $P, \frac{1}{2}$ to $Q$ and 0 to $R$. The valuation st-satisfies $P \Rightarrow Q$ and $Q \Rightarrow R$ and it does not ST-satisfy $P \Rightarrow R$. 


\section{Lowering and (un)labeling in the SK-setting}

As anticipated in Section 5, in the $\mathrm{SK}^{\omega}$-setting the relationship between an inference and its lowering is not as straightforward as in the classical case.

For instance, we know that for any choice of formulas $A, B, C$, the expressions $(A \Rightarrow B),(B \Rightarrow C) \Rightarrow(A \Rightarrow C)$ are tttt-valid 2-inferences, i.e., the notion of tt-satisfaction in a valuation is closed under transitivity (see above Example 6). However, it is not the case that their lowering, that is all 1-inferences of the form $(A \supset B),(B \supset C) \Rightarrow(A \supset C)$ are tt-valid, i.e., in LP the conditional is not transitive (see above Example 2).

At the same time, the quasi-SK-derivation of $(P \supset Q),(Q \supset R) \Rightarrow(P \supset$ $R$ ) in Example 2 can be obtained from the quasi-SK ${ }^{\omega}$-derivation of Example 8 , by uniformly replacing applications of the $L / R \Rightarrow$ rules by applications of the $L / R \supset$ rules, and by replacing labeled inferences of level 1 of the form $A \stackrel{\text { st }}{\Rightarrow} B$ with labeled inferences of level 0 of the form $\mathrm{t}: A \supset B$.

In the case of unsatisfiability, the situation is similar.

These remarks can be made precise and turned into a general analysis of the relationship between $\mathrm{SK}^{\omega}$-notions of satisfaction (and hence of validity and unsatisfiability) of different levels. To do this, we identify the subset of these notions that will be dubbed lowerable. Semantically, we say that a notion of satisfaction for $n$-inferences $\vDash_{I}$ is lowerable iff there is an $\mathrm{SK}^{\omega}$-notion of satisfaction for $(n-1)$ inferences $\vDash_{Y^{\prime}}$ such that for all $n$-inferences $A$ and valuations $v, v \vDash_{I} A$ iff $v \vDash_{I^{\prime}}$ $\operatorname{low}(A)$ (where $\operatorname{low}(A)$ is the lowering of $A$ as we defined it in Section 5).

Remark 19 To avoid possible misunderstanding, we stress that a different notion of lowerability (as a predicate applying to sequents) could be defined by saying that a G3SK ${ }^{\omega}$-sequent $\Gamma \triangleright \Delta$ is lowerable iff there is a set of G3SK-sequents such that the G3SK ${ }^{\omega}$ sequent is satisfied in $v$ iff all G3SK-sequents are. On this understanding of "lowerable", every G3SK ${ }^{\omega}$ sequent is lowerable: by applying the rules of G3SK $^{\omega}$ backwards (as in the construction of a counter-model in the proof of completeness) one can progressively eliminate all higher-level inferences from a given G3SK $^{\omega}$ sequent and thereby obtain a set of SK-sequents which are jointly satisfied iff the given $\mathrm{SK}^{\omega}$-sequent is.

The notion of lowerability we consider (following e.g., [3, 9]) applies to notions of satisfaction, and it is much more restricted than the alternative just considered. In this section, ("our") notion will be used to define the notion of $n$-lowerability (applying again to notions of satisfaction), which applies to those notions of satisfaction for $n$ inferences that can be expressed in terms of the tor s satisfaction of a single formula.

The results about (our notion of) lowerability clearly depend on the chosen logical vocabulary. If the language were expanded with a conditional mirroring ss- or tt-satisfaction of inferences, more notions of satisfaction would be lowerable. Our results however are not ad hoc, since there are very good reasons for not adding such alternative conditionals to the language (in the case of LP, see e.g., [5] and references therein). 
Clearly, for any level $n$, the number of lowerable $\mathrm{SK}^{\omega}$-notions of satisfaction for $n$-inferences can at most be equal to the number of $\mathrm{SK}^{\omega}$-notions of satisfaction for $(n-1)$-inferences. In fact, we show that this is the exact number of lowerable notions, that is for every $\mathrm{SK}^{\omega}$-notion of satisfaction $v \vDash_{1}$ for $n$-inferences, there is one $n+1$ label $I^{\prime}$ such that $v \vDash_{l^{\prime}} A$ iff $v \vDash_{I} \operatorname{low}(A)$ for all $(n+1)$-inferences $A$. We establish these facts by syntactic means.

We begin by explicitly defining the notion of lowerable label and of lowering of a label. ${ }^{19}$ We will then show that for all lowerable I, the notions of I-satisfaction are lowerable in the semantic sense just introduced.

Definition 5 For all levels $n \geq 1$ the notion of lowerable $n$-label and of lowering of a label I, notation low(I), are defined by induction on $n$ as follows:

- $\quad$ st and ts are the lowerable 1-labels, and low(st) = t and low(ts) = s;

- If $I$ and $I^{\prime}$ are lowerable $n$-labels, then $\mathrm{II}^{\prime}$ is a lowerable $n+1$-label, and low $\left(\mathrm{II}^{\prime}\right)=$ low $(\mathrm{I}) \operatorname{low}\left(\mathrm{I}^{\prime}\right)$.

Remark 20 Observe that I being lowerable does not always imply low(I) being lowerable. For instance low(stts) $=$ ts and ts is lowerable, but stst is lowerable and low(stst $)=\mathrm{tt}$ is not lowerable. More on this below.

Theorem 5 For all lowerable n-labels I and n-inferences A:

$$
\vdash_{\mathrm{G}_{3 S K}} \operatorname{low}(\mathrm{I}): \operatorname{low}(A) \triangleright \mathrm{I}: A \quad \vdash_{\mathrm{G}_{3} \mathrm{SK}^{\omega}} \mathrm{I}: A \triangleright \operatorname{low}(\mathrm{I}): \operatorname{low}(A)
$$

Proof By induction on the level of $\mathrm{I}$.

If $I$ is a 0-label, the theorem is obvious (since no 0-label is lowerable).

If $\mathrm{I}$ is a 1-label and $A$ a 1 -inference, say $\Gamma \Rightarrow \Delta$, we have two sub-cases to consider, $\mathrm{I}=\mathrm{st}$ and $\mathrm{I}=\mathrm{ts}$. The theorem is established by observing that derivations of the following four sequents can be obtained by backwards applications of the rules for $\Rightarrow, \supset, \wedge$ and $\vee$ (or $\top$ and $\perp$ if $\Gamma$ and $\Delta$ are empty):

$$
\begin{array}{ll}
\mathrm{t}: \bigwedge \Gamma \supset \bigvee \Delta \triangleright(\Gamma \stackrel{\text { st }}{\Rightarrow} \Delta) & (\Gamma \stackrel{\text { st }}{\Rightarrow} \Delta) \triangleright \mathrm{t}: \bigwedge \Gamma \supset \bigvee \Delta \\
\mathrm{s}: \bigwedge \Gamma \supset \bigvee \Delta \triangleright(\Gamma \stackrel{\text { ts }}{\Rightarrow} \Delta) & (\Gamma \stackrel{\text { ts }}{\Rightarrow} \Delta) \triangleright \mathrm{s}: \bigwedge \Gamma \supset \bigvee \Delta
\end{array}
$$

If $\mathrm{I}$ is a lowerable $n$-label with $n \geq 2$, then $\mathrm{I}=\mathrm{I}^{\prime} \mathrm{I}^{\prime \prime}$ with $\mathrm{I}^{\prime}$ and $\mathrm{I}^{\prime \prime}$ lowerable labels of level $(n-1)$, and $A=\left(B_{1}, \ldots, B_{p} \Rightarrow C_{1}, \ldots, C_{q}\right)$. By induction hypothesis for all $1 \leq i \leq p$ and $1 \leq j \leq q$, there is a derivation of each of the sequents $\mathrm{I}^{\prime}: B_{i} \triangleright \operatorname{low}\left(\mathrm{I}^{\prime}\right): \operatorname{low}\left(B_{i}\right)$ and of each of the sequents $\operatorname{low}\left(\mathrm{I}^{\prime \prime}\right): \operatorname{low}\left(C_{j}\right) \triangleright \mathrm{I}^{\prime \prime}: C_{j}$, from which by an application of $\Rightarrow \mathrm{L}$ (using the admissibility of weakening to obtain derivations sharing the context) followed by one of $\Rightarrow R$ we obtain a derivation of

\footnotetext{
${ }^{19} \mathrm{We}$ will indicate the lowering of a label using the same notation we introduced to indicate the lowering of an inference. The two notions are however distinct, and the different meta-variable for labels and inferences should prevent the confusion between the two notions.
} 
$\operatorname{low}(\mathrm{I}): \operatorname{low}(A) \triangleright \mathrm{I}: A$. A derivation of the other sequent needed to establish the theorem can be obtained in an analogous way.

Corollary 8 If I is lowerable, an inference $\Gamma \Rightarrow \Delta$ is I-valid iff $\operatorname{low}(\Gamma \Rightarrow \Delta)$ is low(I)-valid, and $\Gamma \Rightarrow \Delta$ is I-unsatisfiable iff $\operatorname{low}(\Gamma \Rightarrow \Delta)$ is low(I)-unsatisfiable.

Proof Immediate from Theorem 5 together with Corollary 6 and 7 using the admissibility of cut in $\mathrm{G} \mathrm{SK}^{\omega}$.

This corollary encompasses some recent results concerning specific $\mathrm{SK}^{\omega}{ }^{\omega}$-notions, such as the observation of [2] that ST is LP in sheep's clothing (in our terminology that a 2-inference $A$ is stst-valid iff $\operatorname{low}(A)$ is LP-valid), as well as an analogous correspondence connecting tsts-valid 2-inferences to ss-valid inferences. It is however, far more general, in that, for instance, it entails that the 3-inferences (i.e., metameta-inferences) that are tssttsst-valid are those whose lowerings are the stst-valid 2-inferences (which in turn are those whose lowering are the LP-valid 1-inferences). Moreover, the hierarchy of lowerable notions of satisfaction contributes to clarifying the distinguished role played by the hierarchy of $\mathrm{SK}^{\omega}$-notions of satisfaction introduced by [3] in the $S K^{\omega}$-setting. To this aim we introduce a refinement of the notion of lowerable label, capturing "how many times" a lowerable notion can be lowered:

Definition 6 By induction on $n$ :

- $\quad t$ and $s$ are 0-lowerable;

- $\quad$ if I is lowerable and low $(\mathrm{I})$ is $n$-lowerable then I is $n+1$-lowerable.

A label I is ground lowerable iff it is of level $n$ and it is $n$-lowerable.

Starting from $t$ and $s$ respectively, we define two sequences of labels $\mathbb{T}=$ $t_{0}, t_{1}, t_{2}, \ldots$ and $\mathbb{S}=s_{0}, s_{1}, \ldots$ such that $t_{n}$ and $s_{n}$ are the two ground-lowerable labels of level $n$, for every $n$. The definition relies on the duality function - (see Remark 2) that we define on labels of arbitrary levels in the following way: for 0 -labels, $\overline{\mathrm{s}}=\mathrm{t}$ and $\overline{\mathrm{t}}=\mathrm{s}$; for $n$-labels with $n \geq 1, \overline{\mathrm{l}_{1} \mathrm{l}_{2}}=\mathrm{I}_{2} \mathrm{l}_{1}$.

Definition 7 The sequence of labels $\mathbb{T}=t_{0}, t_{1}, \ldots$ and $\mathbb{S}=s_{0}, s_{1}, \ldots$ are defined as follows:

- $\mathrm{t}_{0}=\mathrm{t}$

- $\quad$ for $n \geq 1, \mathrm{t}_{n}=\overline{\mathrm{t}}_{n-1} \mathrm{t}_{n-1}$

- $\quad \mathrm{s}_{0}=\mathrm{s} ;$
- $\quad$ for $n \geq 1, \mathrm{~s}_{n}=\overline{\mathrm{s}_{n-1}} \mathrm{~s}_{n-1}$

It is easily verified that:

Fact 5 For all $n \geq 1, \operatorname{low}\left(\mathrm{t}_{n}\right)=\mathrm{t}_{n-1}$ and $\operatorname{low}\left(\mathrm{s}_{n}\right)=\mathrm{s}_{n-1}$. 
Hence for all $n, \mathrm{t}_{n}$ and $\mathrm{s}_{n}$ are ground lowerable. It is also not difficult to establish that the notion of validity determined by the $n$-th element of $\mathbb{T}$ coincide with the classical notion of C-validity ${ }^{n}$ for $n$-inferences, and that the no structural $n$-inference ${ }^{20}$ is valid on any of the notion of validity determined by the elements of $\mathbb{S}$ :

Fact $6[3,17]$ For all $n$ and all $n$-inferences $\Gamma \Rightarrow \Delta, \vDash_{\mathrm{t}_{n}} \Gamma \Rightarrow \Delta$ iff $\models^{n} \Gamma \Rightarrow \Delta$.

Proof The sequence $\mathbb{T}$ was introduced by [17] (called there $\mathbf{C M}_{\omega}$ ) and it is referred to by [3] as $\mathbb{S T}$ and by [22] as $\mathfrak{T}$. In all three articles the Fact was shown by semantic means. A syntactic route to the fact arises by combining Fact 5 with Fact 4 and Corollary 2 .

Fact 7 [22] For all $n$, there is no structural inference $\Gamma \Rightarrow \Delta$ such that $\vDash_{\mathrm{s}_{n}} \Gamma \Rightarrow \Delta$.

Proof [22] indicates the sequence $\mathbb{S}$ as $\mathbb{S}$ and proves the fact by semantic means. A syntactic proof follows from Fact 5 together with Corollary 3.

A more direct proof of these two facts can be obtained by scaling the notions of (un)labeling to $\mathrm{GSSK}^{\omega}$. Whereas the notion of unlabeling need no change, we generalize the notion of labeling by replacing in the definition of labeling given in Section 3.2 "formula" with " $n$-inference" and "label" with " $n$-label".

As for G3SK, each derivation $\mathscr{D}$ in $\mathrm{G}_{3} \mathrm{SK}^{\omega}$ is uniquely determined by the $\mathrm{G}_{3} \mathrm{C}^{\omega}$ derivation $u(\mathscr{D})$ and the appropriate labeling function mapping each $n$-inference occurrence in the end-sequent of $u(\mathscr{D})$ onto the label of the corresponding labeled $n$-inference occurrence in the end-sequent of $\mathscr{D}$.

As we did for labels of level 1 , when $l(\Gamma \Rightarrow \Delta)=\mathrm{I}_{1}: \Gamma \Rightarrow \mathrm{I}_{2}: \Delta$, we write $\mathrm{I}_{1} \mathrm{I}_{2}(\Gamma \Rightarrow \Delta)$ for $l(\Gamma \Rightarrow \Delta)$ and similarly $\mathrm{I}_{1} \mathrm{I}_{2}(\mathscr{D})$ for $l(\mathscr{D})$.

Fact 1 scales to $\mathrm{G}_{3} \mathrm{SK}^{\omega}$ in the following way :

Fact 8 If $\mathscr{D}$ is a G3C ${ }^{\omega}$-derivation with end-sequent $\Gamma \triangleright \Delta$ and all elements of $\Gamma$ and $\Delta$ are inferences of the same level $n$, then:

1. $\mathrm{t}_{n}(\mathscr{D})$ is successful;

2. If the end-sequent of $\mathscr{D}$ is structural, then $\mathrm{s}_{n}(\mathscr{D})$ is not successful.

Proof It is easily established by induction that given a G3C-derivation $\mathscr{D}$, in the labeling $\mathrm{t}_{n}(\mathscr{D})$ each $m$-inference $A$ of level $m \leq n$ is labeled by the $m$-th element $\mathrm{t}_{m}$ of $\mathbb{T}$ if $A$ occurs in the succedent, and by the $m$-th element $s_{m}$ of $\mathbb{S}$ if $A$ occurs in the antecedent. Dually, in the labeling $\mathrm{s}_{n}(\mathscr{D})$ each $m$-inference $A$ of level $m \leq n$ is labeled by the $m$-th element $\mathrm{t}_{m}$ of $\mathbb{T}$ if $A$ occurs in the antecedent, and by the $m$-th element $\mathrm{s}_{m}$ of $\mathbb{S}$ if $A$ occurs in the succedent. The fact can thus be proved as Fact 1 and Fact 2.

\footnotetext{
${ }^{20}$ Structural $n$-inference are defined in the same way as structural inferences.
} 
Fact 6 and 7 can thus be proved in the same way as Corollary 2 and 3 above.

\section{Discriminating among Different Notions of Satisfaction}

We finally turn to the issue of showing that all notions of satisfaction, validity and unsatisfiability of a given level $n$ are different from each other. To this aim we define for every level $n$ two sets, $\mathrm{VAL}^{n}$ and $\mathrm{UNS}^{n}$, each containing $2^{n} n$-inferences. We then show that for every label I among the $2^{n+1} n$-labels, I-validity (resp. I-unsatisfiability) is uniquely characterized by one of the subsets of $\mathrm{VAL}^{n}$ (resp. UNS ${ }^{n}$ ), namely the maximum among those whose elements are I-valid (resp. I-unsatisfiable).

We begin by defining the two families of sets $\mathrm{VAL}^{n}$ and $\mathrm{UNS}^{n}$ :

Definition 8 By induction on $n$ :

- $\mathrm{VAL}^{0}=\{A \vee \neg A\}$ and $\mathrm{UNS}^{0}=\{A \wedge \neg A\}$

- For $n>0$ :

$$
\begin{aligned}
& \text { - } \operatorname{VAL}^{n}=\left\{\Rightarrow A \mid A \in \mathrm{VAL}^{n-1}\right\} \cup\left\{A \Rightarrow \mid A \in \mathrm{UNS}^{n-1}\right\} \\
& \text { - } \mathrm{UNS}^{n}=\left\{\Rightarrow A \mid A \in \mathrm{UNS}^{n-1}\right\} \cup\left\{A \Rightarrow \mid A \in \mathrm{VAL}^{n-1}\right\} .
\end{aligned}
$$

Example 9 For $n=2$ we have that

- $\mathrm{VAL}^{2}=\{\Rightarrow(\Rightarrow A \vee \neg A) ; \Rightarrow(A \wedge \neg A \Rightarrow) ;(A \vee \neg A \Rightarrow) \Rightarrow ;(\Rightarrow A \wedge \neg A) \Rightarrow\}$

- $\mathrm{UNS}^{2}=\{\Rightarrow(A \vee \neg A \Rightarrow) ; \Rightarrow(\Rightarrow A \wedge \neg A) ;(\Rightarrow A \vee \neg A) \Rightarrow ;(A \wedge \neg A \Rightarrow) \Rightarrow\}$

In order to establish our result, it is convenient to regard labels as words (i.e., strings of symbols) over the alphabet $\{t, s\}$. From the definition of label (Definition 3 above), it follows immediately that the length $l e n(I)$ of an $n$-label (regarded as a word) is $2^{n}$. For all $n$, let $2^{n}=\left\{m \in \mathbb{N} \mid 1<m \leq 2^{n}\right\}$ be the set of "positions" in a $n$ label. To each $n$-label I there is associated a function which we indicate simply with I (the context always allowing to disambiguate between the label and the associated function), such that I: $\mathbf{2}^{n} \mapsto\{\mathrm{t}, \mathrm{s}\}$ and where $\mathrm{I}(m)$ is the $m$-th symbol of $\mathrm{I}$.

The main idea to establish the result of this section is that for every level $n$ and for every position $m \in 2^{n}$ there is exactly one element of $\mathrm{VAL}^{n}$ (resp. $\mathrm{UNS}^{n}$ ) which is I-valid (resp. I-unsatisfiable) iff $\mathrm{I}(m)$ has a particular value (among $\mathrm{s}$ and $\mathrm{t}$ ) that depends of what we call the polarity of the position $m$. Dwelling on Example 9, for $n=2$ we will show that the first, second, third and fourth 2 -inference in $\mathrm{VAL}^{2}$ above are I-valid iff I is (respectively) of the form xywt, xysz, tywz, xswz (and similarly for unsatisfiability). Thus, the notion of ttss-validity (which is both of the form xysz and tywz but neither of the form xywt nor xswz) is characterized as the only among the 16 notions of validity for 2-inferences on which both the 2-inferences $\Rightarrow(A \wedge \neg A \Rightarrow)$ and $(A \vee \neg A \Rightarrow) \Rightarrow$ but neither $\Rightarrow(\Rightarrow A \vee \neg A)$ nor $(\Rightarrow A \wedge \neg A) \Rightarrow$ are valid.

In order to motivate the notion of polarity, we first observe that, given Definition 4 , if $I_{1} \prec I_{2}$ then $I_{1}$ and $I_{2}$ differ exactly for one symbol, i.e., there is a unique $m$ such that $\mathrm{I}_{1}(m) \neq \mathrm{I}_{2}(m)$ and $\mathrm{I}_{1}\left(m^{\prime}\right)=\mathrm{I}_{2}\left(m^{\prime}\right)$ for all $m^{\prime} \neq m$. Moreover, we observe that whether one obtains a more tolerant or more strict label when one replaces $s$ 
with $\mathrm{t}$ or $\mathrm{t}$ with $\mathrm{s}$ exclusively depends on the position of the replaced symbol in the label. For example (as will be established below in full generality), in the case of 2-labels, whenever $s$ is replaced by $t$ in first and fourth position, and whenever $t$ is replaced by $s$ in second and third position, one obtains a more tolerant notion of validity (respectively, a stricter notion of unsatisfiability).

We spell this out by defining a notion of polarity for positions relative to levels as follows:

Definition 9 For every $n$, the polarity of a position $m$ relative to $n$ is the value of the function pol ${ }^{n}: \mathbf{2}^{n} \mapsto\{+,-\}$ defined by induction on $n$ as follows (where switches + and - , i.e $\overline{\overline{\overline{+}}}=-$ and $\overline{\overline{-}}=+$ ):

- $\operatorname{pol}^{0}(1)=+$

- For $n>0$,

$$
\begin{aligned}
& -\quad \text { if } 1 \leq m \leq \frac{2^{n}}{2} \text { then } \operatorname{pol}^{n}(m)=\overline{\overline{\operatorname{pol}^{n-1}(m)}} \\
& -\quad \text { if } \frac{2^{n}}{2}+1 \leq m \leq 2^{n} \text { then } \operatorname{pol}^{n}(m)=\operatorname{pol}^{n-1}\left(m-\frac{2^{n}}{2}\right)
\end{aligned}
$$

Example 10 The polarity of the positions in 2-labels can be described as follows: $+--+$

As anticipated, the definition is justified by the following fact, that shows that replacing $s$ for $t$ in positive position and $t$ for $s$ in negative positions yields more tolerant labels, whereas replacing $t$ for $s$ in positive position and $s$ for $t$ in negative positions yield stricter labels:

Fact 9 For all $n$-labels $\mathrm{I}_{1}, \mathrm{I}_{2}$, if $\mathrm{I}_{1}(m)=\mathrm{s}, \mathrm{l}_{2}(m)=\mathrm{t}$ and for all $m^{\prime} \neq m \mathrm{I}_{1}\left(m^{\prime}\right)=$ $\mathrm{I}_{2}\left(m^{\prime}\right)$ then

- $\quad \mathrm{I}_{1} \prec \mathrm{I}_{2}$ iff $\mathrm{pol}^{n}(m)=+$

- $\mathrm{I}_{2} \prec \mathrm{I}_{1}$ iff $\operatorname{pol}^{n}(m)=-$

Proof By straightforward induction on $n$ using Definitions 4 and 9.

Remark 21 With reference to Definition 7, for any $n$

- if $\operatorname{pol}^{n}(m)=+$ then $\mathrm{t}_{n}(m)=\mathrm{t}$ and $\mathrm{s}_{n}(m)=\mathrm{s}$

- if $\operatorname{pol}^{n}(m)=-$ then $\mathrm{t}_{n}(m)=\mathrm{s}$ and $\mathrm{s}_{n}(m)=\mathrm{t}$.

From the previous fact and remark, it follows that each label of level $n$ distinct from the one belonging to $\mathbb{S}$ can be reached from this one by successively replacing occurrences of $s$ in positive position with $t$ and occurrences of $t$ in negative positions with s.

The characterization of the different labels of level $n$ using subsets of $\mathrm{VAL}^{n}$ and $\mathrm{UNS}^{n}$ is established with following fact: 
Fact 10 For all $n, m \in \mathbf{2}^{n}$ there is an $A \in \mathrm{VAL}^{n}$ and $B \in \mathrm{UNS}^{n}$ such that for all $n$-labels !:

- if $\operatorname{pol}^{n}(m)=+$ then

$$
\begin{aligned}
& -\vDash_{\mathrm{I}} A \text { iff } \mathrm{I}(m)=\mathrm{t} \text { and } \\
& -\quad B \vDash_{\mathrm{I}} \text { iff } \mathrm{I}(m)=\mathrm{s}
\end{aligned}
$$

- if $\operatorname{pol}^{n}(m)=-$ then

$$
\begin{aligned}
& -\quad \vDash_{\mathrm{I}} A \text { iff } \mathrm{I}(m)=\mathrm{s} \text { and } \\
& -\quad B \vDash_{\mathrm{I}} \text { iff } \mathrm{I}(m)=\mathrm{t}
\end{aligned}
$$

Proof By induction on $n$.

If $n=0,2^{n}=\{1\}, \operatorname{pol}^{n}(1)=+$ and by completeness, the following two derivations and two quasi-derivations verify the fact:

$$
\begin{array}{llll}
\frac{\mathrm{s}: A \triangleright \mathrm{t}: A}{\triangleright \mathrm{t}: A, \mathrm{t}: \neg A} & \frac{\mathrm{t}: A \triangleright \mathrm{s}: A}{\triangleright \mathrm{t}: A \vee \neg A} & \frac{\mathrm{s}: A \triangleright \mathrm{t}: A, \mathrm{~s}: \neg A}{\triangleright \mathrm{s}: A \vee \neg A} & \frac{\mathrm{t}: A \triangleright \mathrm{s}: \neg A \triangleright}{\mathrm{s}: A \wedge \neg A \triangleright}
\end{array} \frac{\mathrm{t}: A, \mathrm{t}: \neg A \triangleright}{\mathrm{t}: A \wedge \neg A \triangleright}
$$

If $n \geq 1$, then for every $n$-label $\mathrm{I}, \mathrm{I}=\mathrm{I}_{1} \mathrm{I}_{2}$ and we consider two cases:

Case 1: $1 \leq m \leq \frac{2^{n}}{2}$. By induction hypothesis, there are $A^{\prime} \in \mathrm{VAL}^{n-1}$ and $B^{\prime} \in$ $\mathrm{UNS}^{n-1}$ satisfying the fact for $m, n-1$ and $\mathrm{I}_{1}$, that is, for every $(n-1)$-label $\mathrm{I}_{1}$ :

- if $\operatorname{pol}^{n-1}(m)=+$ then

- $\operatorname{pol}^{n-1}(m)=-$

$$
\begin{array}{l|l}
-\quad \vDash_{l_{1}} A^{\prime} \text { iff } \mathrm{l}_{1}(m)=\mathrm{t} \text { and } & -\vDash_{\mathrm{l}_{1}} A^{\prime} \text { iff } \mathrm{l}_{1}(m)=\mathrm{s} \text { and } \\
-\quad B^{\prime} \vDash_{\mathrm{l}_{1}} \text { iff } \mathrm{l}_{1}(m)=\mathrm{s} & -B^{\prime} \vDash_{\mathrm{I}_{1}} \text { iff } \mathrm{l}_{1}(m)=\mathrm{t}
\end{array}
$$

The fact is established by taking $A=\left(B^{\prime} \Rightarrow\right)$ and $B=\left(\Rightarrow A^{\prime}\right)$ by observing that there is an $\mathrm{SK}^{\omega}$-derivation of $\mathrm{I}_{1}: B^{\prime} \triangleright$ iff there is one of $\triangleright\left(B^{\prime} \stackrel{\mathrm{I}_{1} \mathrm{I}_{2}}{\Rightarrow}\right)$ and that there is one of $\triangleright \mathrm{I}_{1}: A^{\prime}$ iff there is one of $\left.\stackrel{\mathrm{I}_{1} \mathrm{l}_{2}}{\Rightarrow} A^{\prime}\right) \triangleright$ :

$$
\begin{array}{cc}
\mathscr{D} & \mathscr{D} \\
\frac{\mathrm{I}_{1}: B^{\prime} \triangleright}{\triangleright\left(B^{\prime} \stackrel{\mathrm{I}_{1} l_{2}}{\Rightarrow}\right)} & \frac{\triangleright\left(\mathrm{I}_{1}: A^{\prime}\right)}{\left(\stackrel{\mathrm{l}_{1} \mathrm{l}_{2}}{\Rightarrow} A^{\prime}\right) \triangleright}
\end{array}
$$

The conditions on the labels required in the fact are easily verified keeping in mind that by Definition $9 \mathrm{pol}^{n}(m)=\overline{\overline{\operatorname{pol}^{n-1}(m)}}$.

Case 2: $\frac{2^{n}}{2}+1 \leq m \leq 2^{n}$. By induction hypothesis, there are $A^{\prime} \in \mathrm{VAL}^{n-1}$ and $B^{\prime} \in \mathrm{UNS}^{n-1}$ satisfying the fact for $m-\frac{2^{n}}{2}$ and $n-1$ and $I_{2}$. The fact is verified as in Case 1 by taking $A=\left(\Rightarrow A^{\prime}\right)$ and $B=\left(B^{\prime} \Rightarrow\right)$, where the conditions on the labels required in the fact are easily verified keeping in mind that by Definition 9 $\operatorname{pol}^{n}(m)=\operatorname{pol}^{n-1}\left(m-\frac{2^{n}}{2}\right)$.

In general the question of which are the weakest $\mathrm{SK}^{\omega}$-notions of validity validating a given $\mathrm{C}$-valid $n$-inference $\Gamma \Rightarrow \Delta$ is not obvious. We can answer this question by specifying a simple algorithm using labeling. The algorithm takes as input a G3C ${ }^{\omega}$-derivation $\mathscr{D}$ with end-sequent $\Gamma \triangleright \Delta$ and it tests the validity of the inference according to different $\mathrm{SK}^{\omega}$-notions of validity for $n$-inferences by checking whether the corresponding labelings of $\mathscr{D}$ are successful. More precisely, the algorithm starts by testing whether the labeling corresponding to $s_{n}$ (which we know to 
be the weakest notion, being empty for structural inference) is successul. If it is, then the algorithm stops, otherwise it tests the labelings corresponding to the $n$-notions of validity immediately more tolerant than $s_{n}$. The algorithm proceeds until either all notions still to be tested are more tolerant than one that already gave rise to a successful labeling; or until the last notion to be left is $t_{n}$ (which we know to coincide with the classical notion of validity for $n$-inferences).

\section{Related Work}

As remarked at the beginning of Section 3, the calculus G3SK is a minor modification of the calculi developed by Girard building on previous work by Tait and Schütte. In particular, Girard's calculus has been modified by "absorbing" the structural rules by mixing additive and multiplicative formulations of the rules of each connective. This is a standard technique and the calculi following this pattern are usually labeled "g3" calculi [26], whence the choice of the name for the system here presented. ${ }^{21}$

At the same time, the calculus G3SK is also (a "g3" version of) a particular instance of a general template to obtain sequent calculi for many-valued logics. The proof theory of many-valued logic is a large field of research (see [1] for a survey) and one among the most straightforward approaches to obtain sequent calculi for many-valued logics is the one based on signed-formulas. A signed formula is an expression of the form $w: A$ where $A$ is a formula and $w$ is an element of the set of truth-values $W$. A signed formula $w: A$ is satisfied by a valuation $v$ iff $A$ has value $w$ in $v$. A (one-sided) sequent is a multi-set of signed formulas and it is satisfied by a valuation $v$ if at least one of its elements is satisfied in $v .^{22}$

A sequent calculus for a logic with a semantics based on a finite set of truth values $W=\left\{w_{1}, \ldots, w_{n}\right\}$ can be obtained by associating $n$ distinct rules with each logical constant $\dagger$. For all $1 \leq i \leq n$, the $i$-th rule for a connective allows to "introduce" in the conclusion a signed formula of the form $w_{i}: A$ where $w_{i}$ is the $i$-th element of the set $W$ and $A$ is a logically complex formula governed by $\dagger$. The rules are "read off" from the many-valued truth table of the connective. To give an example,

\footnotetext{
${ }^{21}$ The possibility of absorbing all structural rules relies on the fact that all the rules for the logical connectives are invertible. Whereas this is straightforward in the case of the rule for classical propositional connectives, some care has to be taken in handling the quantifiers or non-classical logics, such as intuitionistic logic. See [26] or [15] for a detailed treatment of these and related issues.

${ }^{22} \mathrm{An}$ inessential alternative arises by considering many-sided sequents instead of multi-sets of signed formulas, where a many-sided sequent is an expression $\Gamma_{1}|\ldots| \Gamma_{n}$, where $n$ is the number of truth-values of the logic in question and $\Gamma_{i}$ is a multiset of formulas for all $0 \leq i \leq n$. A many-sided sequent is said to be satisfied in $v$ if for some $0 \leq i \leq n$ there is an element of $\Gamma_{i}$ with truth value $w_{i}$ in $v$.
} 
consider the truth table describing the assignments of truth-values for conjunctions in SK-valuations:

\begin{tabular}{cc|c}
$A$ & $B$ & $A \wedge B$ \\
\hline 1 & 1 & 1 \\
1 & $1 / 2$ & $1 / 2$ \\
$1 / 2$ & 1 & $1 / 2$ \\
$1 / 2$ & $1 / 2$ & $1 / 2$ \\
1 & 0 & 0 \\
0 & 1 & 0 \\
$1 / 2$ & 0 & 0 \\
$1 / 2$ & 0 & 0 \\
0 & 0 & 0
\end{tabular}

The table tells us that $\llbracket A \wedge B \rrbracket_{v}=1 / 2$ iff the following three conditions are jointly satisfied $\llbracket A \rrbracket_{v}=1$ or $\llbracket A \rrbracket_{v}=1 / 2 ; \llbracket B \rrbracket_{v}=1$ or $\llbracket B \rrbracket_{v}=1 / 2 ; \llbracket A \rrbracket_{v}=1 / 2$ or $\llbracket B \rrbracket_{v}=1 / 2$, which we can turn into the following rule to introduce a conjunction labeled by the truth-value $1 / 2$ :

$$
\frac{\Gamma, 1 / 2: A, 0: A \quad \Gamma, 1 / 2: B, 0: B \quad \Gamma, 1 / 2: A, 1 / 2: B}{\Gamma, 1 / 2: A \wedge B} \wedge 1 / 2
$$

Like in the standard classical case, the table tells us that $\llbracket A \wedge B \rrbracket_{v}=1$ iff both $\llbracket A \rrbracket_{v}=1$ and $\llbracket B \rrbracket_{v}=1$; and that $\llbracket A \wedge B \rrbracket_{v}=0$ iff $\llbracket A \rrbracket_{v}=0$ or $\llbracket B \rrbracket_{v}=0$, yielding two further rules:

$$
\frac{\Gamma, 1: A \quad \Gamma, 1: B}{\Gamma, 1: A \wedge B} \quad \frac{\Gamma, 0: A, 0: B}{\Gamma, 0: A \wedge B}
$$

In the mid-90s several authors (see among others [13, 21, 27]) realized that "optimized" sequent calculi (i.e., calculi with fewer rules and/or fewer branching in some rules) could be obtained by labeling formulas with sub-sets (instead of elements) of the set of truth-values (so that a multi-set of labeled formulas $W_{1}: A_{1}, \ldots, W_{m}: A_{m}$ is satisfied in $v$ iff there is an $1 \leq i \leq m$ such that the value of $A_{i}$ in $v$ is included in $\left.W_{i}\right)$. The calculus G3SK can be seen as a two-sided variant of such calculi with $t$ and $\mathrm{s}$ being short for $\{1 / 2,1\}$ and $\{1\}$.

The generalization of G3SK to G3SK ${ }^{\omega}$ by the addition of inferences of higher-level suggests a connection with at least another strand of research, this time in the prooftheory of modal logic. In order to obtain well-behaved sequent calculi for different propositional modal logics, the notion of sequent has been generalized by allowing some form of nesting of sequents inside sequents (different authors introduced this idea independently and under different names, see $[4,10,14,18])$. What is common to the different formulations of nested sequent calculi is the possibility of applying the operational rules (i.e., the rules introducing logical connectives in the antecedent or succedent of sequents) at different "depths". Although our calculus combines nesting of inferential arrows and labels, some "nested versions" of some inference rules (namely all those in which the principal formulas occur on the same side of the sequent separator) are admissible in G3SK. For instances, it is not difficult to show 
that the following rules are admissible in $\mathrm{G}_{3} \mathrm{SK}^{\omega}$ :

$$
\frac{[A, B] \Gamma \triangleright \Delta}{[A \wedge B] \Gamma \triangleright \Delta} \mathrm{L} \wedge \text {-nested } \quad \frac{\Gamma \triangleright \Delta[A] \quad \Gamma \triangleright \Delta[B]}{\Gamma \triangleright \Delta[A \wedge B]} \mathrm{R} \wedge \text {-nested }
$$

(where the left rule is to be understood as allowing the replacement of one occurrence of $A$ and of one occurrence of $B$ with one of $A \wedge B$, provided the occurrences of $A$ and of $B$ figure on the left of the arrow symbol of a 1 -inference in $\Gamma$; and the right rule is to be understood so that its premises are two copies of the same sequent in which one occurrence of $A$ to the right of the arrow symbol of a 1 -inference in $\Delta$ has been replaced by $B$, and whose conclusion is obtained from one of the premises by replacing the indicated occurrence of $A$ or $B$ with $A \wedge B$ ): As the reader can easily verify, whenever, e.g., $((A, B, \Gamma \Rightarrow \Delta), \Sigma \stackrel{\text { xywz }}{\Rightarrow} \Theta), \Phi \triangleright \Psi$ is derivable, so is $((A \wedge B, \Gamma \Rightarrow \Delta), \Sigma \stackrel{\text { xywz }}{\Rightarrow} \Theta), \Phi \triangleright \Psi$.

Analogous nested versions of the rules for $\neg$ and $\supset$ are not admissible, due to the fact that the rules involve shifting formulas from one side to the other of inferences. For example, it is not the case that if $((A, \Gamma \Rightarrow \Delta, B), \Sigma \stackrel{\text { xywz }}{\Rightarrow} \Theta), \Phi \triangleright \Psi$ is derivable then $((\Gamma \Rightarrow \Delta, A \supset B), \Sigma \stackrel{\text { xywz }}{\Rightarrow} \Theta), \Phi \triangleright \Psi$ is derivable as well, at least not for every choice of $\mathrm{x}$ and $\mathrm{y}$. This is due to the fact that in order to introduce an implication to the right of a sequent its sub-formulas in the premises must have distinct labels and this induces certain restrictions on the possibility of applying a nested version of the rules (in the example just given, $x$ and $y$ must be distinct).

This of course does not exclude the possibility of formulating appropriate restrictions on nested versions of the rules that may yield a proof system in which complex formulas can be introduced even inside nested inferences. We leave the exploration of this direction of investigation (and related ones, see below in Section 11) for future work.

We conclude this section by stressing that although all elements of the proof system G3SK ${ }^{\omega}$ introduced in the paper have appeared in some form or another in the literature, the way in which we combined these elements is new. Although the results presented are not particularly difficult and could have been worked out using other background systems, we believe that they allow a smooth reconstruction of several results concerning the SK-setting that have appeared in piecemeal form in the recent literature. Moreover, most of the above mentioned works in the proof theory of many valued and modal logic have focused on the generality of the results (in particular applicability to the widest range of logical system possible) with the consequence of making them less accessible for the more philosophically oriented community interested in the classes of logics that arise in the SK-setting.

\section{Concluding Philosophical Remarks}

Implicit in the philosophical discussions of early results about the SK-setting, and explicitly argued by [3], is the claim that a "logic" is determined neither by a notion of validity for formulas, nor by a notion of validity for formulas and inferences: The set of classically valid formulas, of LP-valid formulas and of ST-valid formulas 
coincide. In the case of ST, not even looking at the validity of inferences is enough to distinguish it from classical logic. Thus—-so argue Barrio, Pailos and Szmuc—a logic should be determined by laying down neither one nor two notions of satisfaction, but a countable sequence of notions of satisfaction, one for each level $n$ of the hierarchy arising in the SK setting.

A natural question that these considerations suggest is whether any sequence of countably many labels $\left\langle\mathrm{I}_{0}, \mathrm{I}_{1}, \ldots\right\rangle$ (such that $\mathrm{I}_{i}$ is a label of level $i$ for all $i \geq 0$ ) can be taken to determine a logic. Or should one rather say that only sequences satisfying some additional conditions really determine a logic?

It seems that two distinct conditions have been implicitly acknowledged as being sufficient for a sequence of labels to identify a logic.

The first condition could be dubbed uniformity. Uniformity is the requirement that in the sequence of notions determining a logic, the $n$-th notion of satisfaction in the sequence is obtained by evaluating both antecedent and succedent of inferences according to the standards of the $(n-1)$-th notion of satisfaction in the sequence. Uniformity is implicitly endorsed when one identifies e.g., LP-satisfaction of formulas, inferences (and more recently "meta-inferences") with t-, tt- and tttt-satisfaction respectively.

The recent interest in "mixed" notions of satisfaction (such as e.g., st-satisfaction of inferences) suggests that uniformity should not be regarded as a necessary condition for a sequence of labels to constitute a logic. Remarkably, however, even those authors who advocated a "mixed" notion of satisfaction for inferences, implicitly endorsed uniformity as the criterion to choose the notion of satisfaction for 2inferences, as testified by the fact that "st-satisfaction of a meta-inference" is usually taken to mean "stst-satisfaction" (see Remark 15 above).

Nonetheless, by seriously adopting uniformity as the guiding principle to define a sequence of notions of satisfaction, one would end up with only two possible "logics" in the SK-setting, namely those identified by the two sequences of labels $\langle t, t t, t t t, \ldots\rangle$ and $\langle\mathrm{s}, \mathrm{ss}, \mathrm{ssss}, \ldots\rangle$.

Underlying the criterion of uniformity is the idea that the higher-level notions of satisfaction conceptually depend on the lower ones. This is not the only way to look at the relationship between lower-level and higher-level notions of satisfaction, but it is certainly very plausible. It is true that any notion of satisfaction of level $n+1$ automatically determines a notion of satisfaction of level $n^{23}$, and thus that lower-level notions are dependent on the higher-level ones. However, the notions of lower level are motivated by certain intuitions which we seem to lack for higher-level notions (at least, as soon as one goes beyond level 2, but see more on this below) and this might be taken as a reason for assigning conceptual priority to the former ones.

At any rate, there seem to be different ways to express the conceptual dependency of higher-level notions on lower-level ones. Beside uniformity, another possibility is offered by the notion of lowerability we discussed in Section 8 . The sequences of notions of satisfaction described by the sequences of labels $\mathbb{T}=\left\langle\mathrm{t}_{0}, \mathrm{t}_{1}, \ldots\right\rangle$ and $\mathbb{S}=\left\langle\mathrm{s}_{0}, \mathrm{~s}_{1}, \ldots\right\rangle$ are constituted by labels which are all ground lowerable, that is the

\footnotetext{
${ }^{23}$ An inference $(\Rightarrow A)$ is $\mathrm{I}_{1} \mathrm{l}_{2}$-satisfied by $v$ iff $A$ is $\mathrm{I}_{2}$-satisfied by $v$.
} 
$\mathrm{t}_{n}$ - $\left(\right.$ resp. $\left.\mathrm{s}_{n}\right)$-satisfaction of an $n$-inference in a valuation $v$ can be expressed as the $\mathrm{t}-$ (resp. s)-satisfaction of a formula in $v$.

Also in this case, however, by taking lowerability as the guiding principle to define a sequence of notions of satisfaction, one would end up with only two possible "logics" in the SK-setting, namely those identified by the two sequences of labels $\mathbb{T}$ and $\mathbb{S}$.

It is worth stressing that the two conditions of uniformity and lowerability are at odds in the SK-setting, so that there is no sequence of notions of satisfaction that complies with both. In contrast, the hierarchy of classical notions satisfies both uniformity and lowerability. Therefore, anyone who is willing to acknowledge uniformity and lowerability as "desirable features" of a logic, must accord to classical logic (understood as the sequence of classical notions of satisfaction for all inferential levels) a distinguished status over any of the "logics" arising by any sequence of notions of satisfaction in the SK-setting.

In order to argue against the idea that either uniformity or lowerability play any distinctive role in characterizing a logic as a sequence of notions of satisfaction, the advocate of the SK-setting should provide some intuitions for the plethora of different notions of satisfaction that we encounter on each level of the SK-setting. Whereas this has been done for the notion of st-satisfaction (and to some extent, for that of tssatisfaction), nothing has been said so far of all notions of higher-level (except those of level 2, see [16] and the few ones which could be justified by appealing to either uniformity or lowerability).

We conclude this section by suggesting a possible reading of higher-level inferences which could provide the basis for developing some intuitions about at least some of the higher-level notions of satisfaction in the SK-setting. According to a certain formulation of bilateralism (see e.g., [19]), an inference $\Gamma \Rightarrow \Delta$ expresses the inconsistency of accepting all formulas in the antecedent and rejecting all those in the succedent. ${ }^{24}$ The four SK-notions of validity of an inference can be distinguished considering the inferences $A \wedge \neg A \Rightarrow$ and $\Rightarrow A \vee \neg A$, i.e. according to whether they take it to be inconsistent to accept $A \wedge \neg A$ or to reject $A \vee \neg A$ (on st-standards both are inconsistent; on ss-standards the former but not the latter is inconsistent; on tt-standards the latter but not the former is inconsistent, and on ts-standard neither is inconsistent).

The bilateral reading of inferences generalizes straightforwardly to inferences of higher-level, so that e.g., the 2-inference $\Rightarrow(\Rightarrow A)$ expresses that it is inconsistent to reject that it is inconsistent to reject $A$, and the 2-inference $(\Rightarrow A) \Rightarrow$ expresses that it is inconsistent to accept that it is inconsistent to reject $A$, and so on.

From a bilateral perspective, paraconsistency and paracompleteness can be understood as general features of the notions of acceptance and rejection of propositions, so that paraconsistency is the claim that for some proposition $A$ it is not inconsistent to accept both it and its negation, and paracompleteness is the thesis that for some proposition $A$ it is not inconsistent to reject both it and its negation. By

\footnotetext{
${ }^{24} \mathrm{We}$ choose to speak of acceptance and rejection rather than assertion and denial, and thereby to focus on subjects' attitudes towards propositions rather than on the linguistic manifestations of these attitudes by means of speech acts.
} 
considering higher-level inferences, paraconsistency and paracompleteness can be scaled to acceptance and rejection "of higher-level". At level 2, we might speak of 2paraconsistency and 2-paracompleteness, where each comes in two possible forms: 2-paraconsistency can be equated with endorsing either of the following two thesis (or both):

- it is not inconsistent to accept that it is inconsistent to reject both $A$ and its negation (such a thesis corresponds to the non-validity of the 2-inference ( $\Rightarrow$ $A \wedge \neg A) \Rightarrow$;

- it is not inconsistent to accept that it is inconsistent to accept either $A$ or its negation (such a thesis corresponds to the non-validity of the 2-inference ( $A \vee$ $\neg A \Rightarrow) \Rightarrow)$;

Although the formulations are pretty involved already at level 2, they suggest the possibility of drawing fine-grained distinctions between more and less permissive understanding of the (higher-level) attitudes of acceptance and rejection of the inconsistency of combinations of lower-level acceptance and rejection. The sets of inferences $\mathrm{VAL}^{n}$ and $\mathrm{UNS}^{n}$ introduced in Section 9 to discriminate among the different notions of satisfaction at each level suggest a general pattern for describing the different forms that paraconsistency and paracompleteness might take at each level.

Finally, we remark that the notion of higher-level inference might also be endowed with a modal flavor (by therefore thinking of $\Rightarrow$ as a sort of higher-level strict conditional rather than material conditional). Although the formal development of this idea would require substantial changes to the calculus G3SK ${ }^{\omega}$ (e.g., by applying appropriate restrictions on the contexts of the rules $\Rightarrow R$ and $\Rightarrow L$ ), the resulting setting might offer a more plausible reading of higher-level inference. In particular, the properties of higher-level inferences and the relationship between different levels could be investigated using tools developed in the study of modal logic (in particular, the study of different properties of higher-level inferences might be captured using syntactic devices mimicking the different frame conditions underlying modalized conditionals).

This and further directions of investigation will be explored in further work.

Acknowledgements The authors wish to thank the referees for detailed and useful comments on previous versions of the paper. Luca Tranchini acknowledges the Deutsche Forschungsgemeinschaft for funding his research as part of the project "Falsity and Refutations. Understanding the negative side of logic" (DFG: TR1112/4-1). This research was also supported by the Ministry of Science, Innovation and Universities of the Government of Spain with the project "Logic and Substructurality (FFI2017-84805-P).

Funding Open Access funding enabled and organized by Projekt DEAL.

Open Access This article is licensed under a Creative Commons Attribution 4.0 International License, which permits use, sharing, adaptation, distribution and reproduction in any medium or format, as long as you give appropriate credit to the original author(s) and the source, provide a link to the Creative Commons licence, and indicate if changes were made. The images or other third party material in this article are included in the article's Creative Commons licence, unless indicated otherwise in a credit line to the material. If material is not included in the article's Creative Commons licence and your intended use is not permitted by statutory regulation or exceeds the permitted use, you will need to obtain permission directly from the copyright holder. To view a copy of this licence, visit http://creativecommons.org/licenses/by/4.0/. 


\section{References}

1. Baaz, M., Fermüller, C. G., \& Salzer, G. (2001). Automated deduction for Many-Valued logics. In J. A. Robinson, \& A. Voronkov (Eds.) Handbook of automated reasoning, (Vol. II pp. 1355-1402). Elsevier.

2. Barrio, E., Rosenblatt, L., \& Tajer, D. (2015). The logics of strict-tolerant logic. Journal of Philosophical Logic, 44(5), 551-571.

3. Barrio, E. A., Pailos, F., \& Szmuc, D. (2020). A hierarchy of classical and paraconsistent logics. Journal of Philosophical Logic, 49, 93-120.

4. Brünnler, T. (2006). Deep sequent systems for modal logic'. Advances in Modal Logic AiML, 6, 107-119.

5. Carrara, M., \& Martino, E. (2014). Logical consequence and conditionals from a dialethic perspective. Logique \& Analyse, 227, 359-378.

6. Cobreros, P., Égré, P., Ripley, D., \& van Rooij, R. (2012). Tolerant, classical, strict. Journal of Philosophical Logic, 41(2), 347-385.

7. Cobreros, P., La Rosa, E., \& Tranchini, L. (2020). (I Can't get no) antisatisfaction. Synthese, Online First. https://doi.org/10.1007/s11229-020-02570-x.

8. Da Ré, B., Szmuc, D., \& Teijeiro, P (2021). Derivability and Metainferential Validity. Journal of Philosophical Logic, Online First. https://doi.org/10.1007/s10992-021-09619-3.

9. Dicher, B., \& Paoli, F. (2019). ST, LP, and tolerant metainferences. In C. Baskent, \& T. M. Ferguson (Eds.) Graham priest on dialetheism and paraconsistency. Outstanding contributions to logic, (Vol. 18 pp. 383-407). Springer.

10. Došen, K. (1980). Logical constants: An essay in proof theory, University of Oxford, Oxford.

11. French, R. (2016). Structural reflexivity and the paradoxes of self-reference. Ergo, 3(5), 113-131.

12. Girard, J.-Y. (1987). Proof theory and logical complexity, Volume 1. Bibliopolis.

13. Haehnle, R. (1993). Automated deduction in multiple-valued logics. Oxford: Clarendon Press.

14. Kashima, R. (1994). 'Cut-free sequent calculi for some tense logics'. Studia Logica, 53, 119-135.

15. Negri, S., Von Plato, J., \& Ranta, A. (2008). Structural proof theory. Cambridge: Cambridge University Press.

16. Pailos, F. (2019). A family of metainferential logics. Journal of Applied Non-Classical Logics, 29(1), 97-120.

17. Pailos, F. (2019b). A fully classical truth theory characterized by substructural means. The Review of Symbolic Logic, 13(2), 249-268.

18. Poggiolesi, F. (2008). The method of tree-hypersequents for modal propositional logic. In D. Makinson, J. Malinowski, \& H. Wansing (Eds.) Towards mathematical philosophy (pp. 31-51). Springer.

19. Ripley, D. (2013). Paradoxes and failures of cut. Australasian Journal of Philosophy, 91(1), 139-164.

20. Rousseau, G. (1967). Sequents in many valued logic I. Fundamenta Mathematicae, 60(1), 23-33.

21. Salzer, G. (1996). Optimal axiomatizations for multiple-valued operators and quantifiers based on semi-lattices. In M. A. McRobbie, \& J. K. Slaney (Eds.) Automated deduction-cade-13. CADE 1996. Lecture notes in computer science (lecture notes in artificial intelligence), (Vol. 1104 pp. 688-702). Berlin: Springer.

22. Scambler, C. (2020a). Classical logic and the strict tolerant hierarchy. Journal of Philosophical Logic, 49, 351-370.

23. Scambler, C. (2020). Transfinite meta-inferences. Journal of Philosophical Logic, 49, 1079-1089.

24. Schroeder-Heister, P. ((1984). A natural extension of natural deduction. The Journal of Symbolic Logic, 49(4), 1284-1300.

25. Schröter, K. (1955). Methoden zur Axiomatisierung beliebiger Aussagen- und prädikatenkalküle. Mathematical Logic Quarterly, 4(1), 241-251.

26. Troelstra, A. S., \& Schwichtemberg, H. (1996). Basic proof theory. Cambridge: Cambridge University Press.

27. Zach, R. (1993). Proof theory of finite-valued logics. Technische Universität Wien: Diplomarbeit.

Publisher's Note Springer Nature remains neutral with regard to jurisdictional claims in published maps and institutional affiliations. 